\title{
Generation of Phosphorus-Centered Radicals via Homolytic Substitution at Sulfur
}

\author{
Paola Carta, Nicolas Puljic, Carine Robert, Anne-Lise Dhimane, Louis Fensterbank, ${ }^{*}$ \\ Emmanuel Lacôte, * Max Malacria*
}

\section{Supporting information}

General remarks: Reagents and chemicals were purchased from commercial sources and used as received. Reactions were carried out under argon, with magnetic stirring and degassed solvents. $\mathrm{CH}_{2} \mathrm{Cl}_{2}$, benzene and toluene were dried and distilled from $\mathrm{CaH}_{2}$. Thin-layer chromatography (TLC) was performed on Merck 60 F254 silica gel. Merck Geduran SI 60 A silica gel (35-70 mm) was used for column chromatography. The melting points reported were measured with a Reichert hot-stage apparatus and are uncorrected. IR spectra were recorded from a Bruker Tensor 27 ATR diamond PIKE spectrometer. ${ }^{1} \mathrm{H}$ NMR $\left[{ }^{13} \mathrm{C}\right.$ NMR $]$ spectra were recorded at room temperature with $200 \mathrm{MHz}$ [50 MHz] Bruker ARX 200 and $400 \mathrm{MHz}$ [100 MHz] Bruker AVANCE 400 spectrometers. Chemical shifts are given in ppm, referenced to the residual proton resonances of the solvents $(\delta=$ 7.28 or 77.16 , respectively, for $\left.\mathrm{CDCl}_{3}\right) .{ }^{31} \mathrm{P} \mathrm{NMR}$ was recorded with $162 \mathrm{MHz}$ Bruker ARX 400 and AVANCE 400 spectrometers. Chemical shifts are given in ppm, referenced to the external phosphorus resonance of $85 \%$ aq. $\mathrm{H}_{3} \mathrm{PO}_{4}(0 \mathrm{ppm})$. Coupling constants $(J)$ are given in Hertz $(\mathrm{Hz})$. Elemental analysis were performed by the Service Régional de Microanalyse de L'Université Pierre et Marie Curie and by the ICSN (CNRS, Gif).

\section{GP1: Preparation of the substrates}

To the starting thiol $(5 \mathrm{mmol})$ in THF $(30 \mathrm{~mL})$ was added $\mathrm{NaH}(60 \%$ in mineral oil; $6 \mathrm{mmol}$; 1.1 equiv.; $240 \mathrm{mg})$ at $0^{\circ} \mathrm{C}$. The $\mathrm{R}_{2} \mathrm{P}(=\mathrm{O}) \mathrm{Cl}$ derivative $(6 \mathrm{mmol} ; 1.1$ equiv.) was added after $5 \mathrm{~min}$ and the reaction was left at r.t. until completion, as monitored by TLC. After completion, the reaction mixture was diluted with $\mathrm{Et}_{2} \mathrm{O}(15 \mathrm{~mL})$, washed with sat. aq. $\mathrm{NH}_{4} \mathrm{Cl}$, water, and brine. The organic phase was dried over $\mathrm{MgSO}_{4}$, filtered, and concentated in vacuo. The crude product was purified by flash column chromatography (silicagel).

\section{GP2: tributyl hydride-mediated formation of C-P bonds}

A degassed solution of AIBN ( $0.12 \mathrm{mmol} ; 0.15$ equiv; $15 \mathrm{mg})$ and $\mathrm{Bu}_{3} \mathrm{SnH}(0.6 \mathrm{mmol}$; 1.2 equiv.; $0.16 \mathrm{ml})$ in toluene $(5 \mathrm{mmol})$ was added via syringe pump $\left(0.2 \mathrm{mmol}^{-1}\right)$ to a refluxed and degassed solution of the thiophosphonyl derivative $(0.5 \mathrm{mmol} ; 1$ equiv. $)$, the alkene $(5 \mathrm{mmol} ; 10$ equiv. $)$, and AIBN (0.04 mmol; 0.05 equiv.; $5 \mathrm{mg}$ ) in toluene $(12 \mathrm{~mL})$. After completion of the addition, the reaction was refluxed an additional hour, and concentrated. Flash column chromatography (silicagel) delivered the expected product.

\section{GP3: Catalytic conditions}

A degassed solution of the thiophosphonyl derivative $(0.5 \mathrm{mmol} ; 1$ equiv. $), \mathrm{Bu}_{3} \mathrm{SnCl}(0.06 \mathrm{mmol}$; 0.1 equiv.; $0.16 \mathrm{ml}), \mathrm{NaBH}_{4}$ ( $1 \mathrm{mmol} ; 2$ equiv.; $\left.38 \mathrm{mg}\right)$, the alkene ( $1 \mathrm{mmol}$; 2 equiv.), AIBN $(0.05 \mathrm{mmol}$; 0.10 equiv.; $9 \mathrm{mg})$ in $t$-butanol $(25 \mathrm{ml})$ was refluxed for 5 hours (AIBN (0.05 mmol; 0.10 equiv.; $9 \mathrm{mg}$ ) was added again after 2.5 hour). After completion, the reaction mixture was treated with sat. aq. $\mathrm{NH}_{4} \mathrm{Cl}$. The aqueous phase was extracted with $\mathrm{Et}_{2} \mathrm{O}(3 \times 15 \mathrm{ml})$. The combined organics were washed with brine, dried over $\mathrm{MgSO}_{4}$, filtered, and concentated in vacuo. The crude product was purified by flash column chromatography (silicagel). 
<smiles>O=P(SCCc1ccccc1Br)(c1ccccc1)c1ccccc1</smiles>

1a: Following GP1, substrate 1a was isolated (petroleum ether/ethyl acetate $2: 1,1.711 \mathrm{~g}, 82 \%$ ) as a colorless dense oil. IR (neat): 3056, 2927, 1589, 1568, $1113 \mathrm{~cm}^{-1} ;{ }^{1} \mathrm{H} \mathrm{NMR}\left(\mathrm{CDCl}_{3}, 400 \mathrm{MHz}\right): \delta$ 3.05-3.10 (m, $4 \mathrm{H}, \mathrm{CH}_{2} \mathrm{CH}_{2}$ ), 7.05-7.10 (m, $1 \mathrm{H}$ arom.), 7.16-7.25 (m, $2 \mathrm{H}$ arom.), 7.47-7.60 (m, $7 \mathrm{H}$ arom.), 7.88-7.92 (m, $4 \mathrm{H}$ arom.); ${ }^{13} \mathrm{C} \mathrm{NMR}\left(\mathrm{CDCl}_{3}, 100 \mathrm{MHz}\right): \delta 28.6(\mathrm{~d}, J=2.2 \mathrm{~Hz}, \mathrm{ArCH}), 37.3$ (d, $\left.J=4.4 \mathrm{~Hz}, \mathrm{SCH}_{2}\right), 124.3$ (C arom.), 127.5 (CH arom.), 128.4 (CH arom.), 128.7 (d, $J=10.3 \mathrm{~Hz}$, $\mathrm{CH}$ arom.), 131.1 (CH arom.), 131.5 (d, $J=10.3 \mathrm{~Hz}, \mathrm{CH}$ arom.), 132.4 (d, $J=2.6 \mathrm{~Hz}, \mathrm{CH}$ arom.), 132.9 (CH arom.), 133.2 (d, $J=106.2 \mathrm{~Hz},=\mathrm{CP}), 138.7$ (C arom.); ${ }^{31} \mathrm{P}$ NMR $\left(\mathrm{CDCl}_{3}, 162 \mathrm{MHz}\right): \delta$ 44.6; elemental analysis (\%) for $\mathrm{C}_{20} \mathrm{H}_{18} \mathrm{BrOPS}$ (417.30): calcd. C 57.56, H 4.35; found C 57.12, $\mathrm{H} 4.43$; HRMS calcd. for $\mathrm{C}_{20} \mathrm{H}_{18}{ }^{81} \mathrm{BrONaPS}\left([\mathrm{M}+\mathrm{Na}]^{+}\right)$: 440.9877 , found 440.9840 .

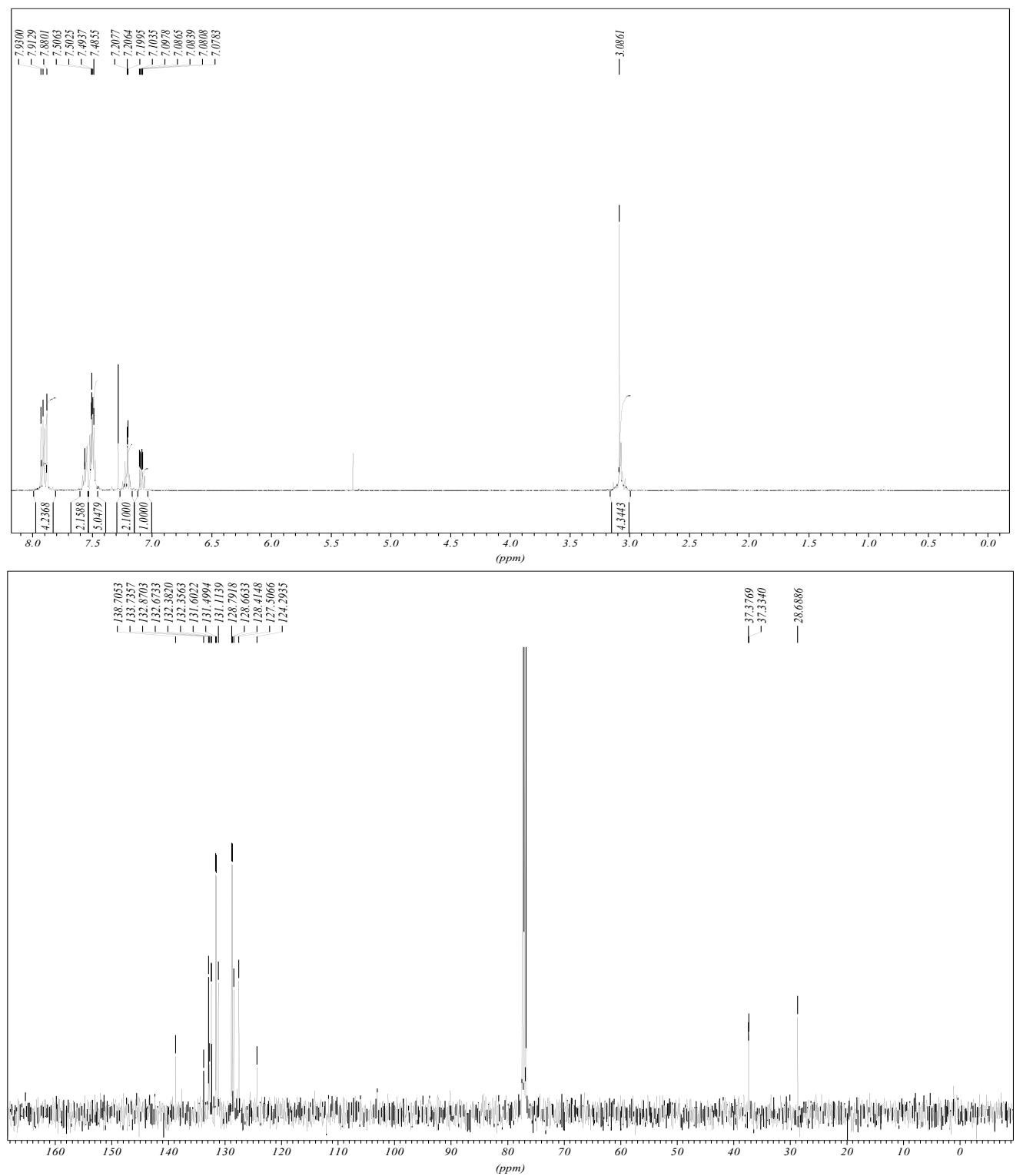


<smiles>CCOP(=O)(OCC)SCCc1ccccc1Br</smiles>

1b: Following GP1, substrate 1b was isolated (petroleum ether/ethyl acetate 2:1, 1.166 g, 66\%) as a colorless oil. IR (neat): 2981, 2927, $1010 \mathrm{~cm}^{-1} ;{ }^{1} \mathrm{H} \mathrm{NMR}\left(\mathrm{CDCl}_{3}, 400 \mathrm{MHz}\right): \delta 1.33$ (t, $J=7.1 \mathrm{~Hz}$, $\left.6 \mathrm{H}, \mathrm{OCH}_{2} \mathrm{Me}\right)$, 2.99-3.11 (m, $\left.4 \mathrm{H}, \mathrm{CH}_{2} \mathrm{CH}_{2}\right), 4.06-4.20\left(\mathrm{~m}, 4 \mathrm{H}, \mathrm{OCH}_{2} \mathrm{Me}\right), 7.04-7.10(\mathrm{~m}, 1 \mathrm{H}$

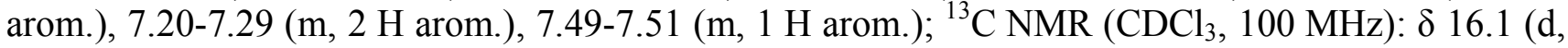
$\left.J=7.7 \mathrm{~Hz}, \mathrm{OCH}_{2} \mathrm{Me}\right), 30.1\left(\mathrm{ArCH}_{2}\right), 37.4\left(\mathrm{~d}, J=5.1 \mathrm{~Hz}, \mathrm{SCH}_{2}\right), 63.5\left(\mathrm{~d}, J=5.1 \mathrm{~Hz}, \mathrm{OCH}_{2} \mathrm{Me}\right)$, 124.3 (C arom.), 127.6 (CH arom.), 128.5 ( $\mathrm{CH}$ arom.), 131.0 (CH arom.), 132.9 (CH arom.), 138.6 (C arom.); ${ }^{31} \mathrm{P} \mathrm{NMR}\left(\mathrm{CDCl}_{3}, 162 \mathrm{MHz}\right): \delta 28.9$; elemental analysis (\%) for $\mathrm{C}_{12} \mathrm{H}_{18} \mathrm{BrO}_{3} \mathrm{PS}(353.21)$ : calcd. C 40.81, H 5.14; found C 40.65, H 5.26; HRMS calcd. for $\mathrm{C}_{12} \mathrm{H}_{18}{ }^{81} \mathrm{BrO}_{3} \mathrm{NaPS}\left([\mathrm{M}+\mathrm{Na}]^{+}\right)$: 376.9775 , found 376.9764 .

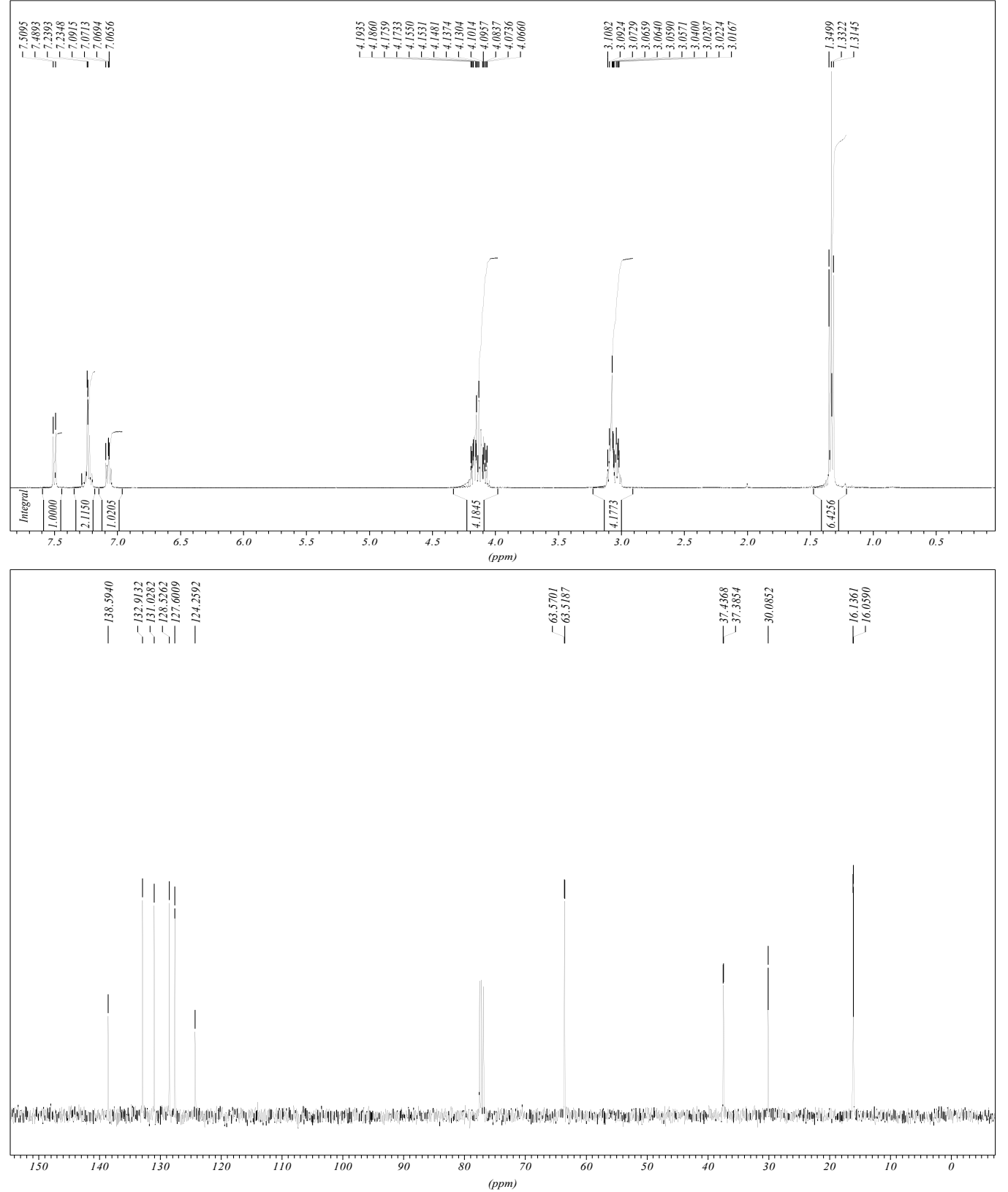


<smiles>CN1CCN(C)P1(=O)SCCc1ccccc1Br</smiles>

1c: Following GP1, substrate 1c was isolated (petroleum ether/ethyl acetate 1:1, $978 \mathrm{mg}, 56 \%$ ) as a whitish dense oil. IR (neat): 2986, 2928, $1159 \mathrm{~cm}^{-1} ;{ }^{1} \mathrm{H} \mathrm{NMR}\left(\mathrm{CDCl}_{3}, 400 \mathrm{MHz}\right): \delta 7.56(\mathrm{~d}, J=8.3$, 1H), 2.68 (s, $3 \mathrm{H}, \mathrm{NMe}), 2.71$ (s, $3 \mathrm{H}, \mathrm{NMe}), 2.86-2.93$ (m, $\left.2 \mathrm{H}, \mathrm{CH}_{2}\right), 3.05-3.08$ (m, $\left.2 \mathrm{H}, \mathrm{CH}_{2}\right), 3.12-$ 3.18 (m, $\left.2 \mathrm{H}, \mathrm{CH}_{2}\right), 3.30-3.36$ (m, $\left.2 \mathrm{H}, \mathrm{NCH}_{2}\right), 7.10-7.14$ (m, $1 \mathrm{H}$ arom.), 7.25-7.29 (m, $2 \mathrm{H}$ arom.), 7.54-7.56 (m, $1 \mathrm{H}$ arom.); ${ }^{13} \mathrm{C} \mathrm{NMR}\left(\mathrm{CDCl}_{3}, 100 \mathrm{MHz}\right): \delta 30.2$ (d, $\left.J=4.3 \mathrm{~Hz}, \mathrm{CH}_{2} \mathrm{Ar}\right), 31.3$ (d, $J=6.0 \mathrm{~Hz}, \mathrm{NMe}), 37.6\left(\mathrm{~d}, J=5.1 \mathrm{~Hz}, \mathrm{CH}_{2} \mathrm{~S}\right), 47.6\left(\mathrm{~d}, J=9.4 \mathrm{~Hz}, \mathrm{NCH}_{2}\right), 124.2$ (C arom.), 127.6 (CH arom.), 128.4 (CH arom.), 131.1 ( $\mathrm{CH}$ arom.), 132.9 (CH arom.), 139.1 (C arom.); ${ }^{31} \mathrm{P}$ NMR $\left(\mathrm{CDCl}_{3}, 162 \mathrm{MHz}\right): \delta$ 42.1; elemental analysis (\%) for $\mathrm{C}_{12} \mathrm{H}_{18} \mathrm{BrN}_{2} \mathrm{OPS}$ (349.23): calcd. C 41.27, H 5.20, N 8.02; found C 40.94, H 5.22, N 7.94.
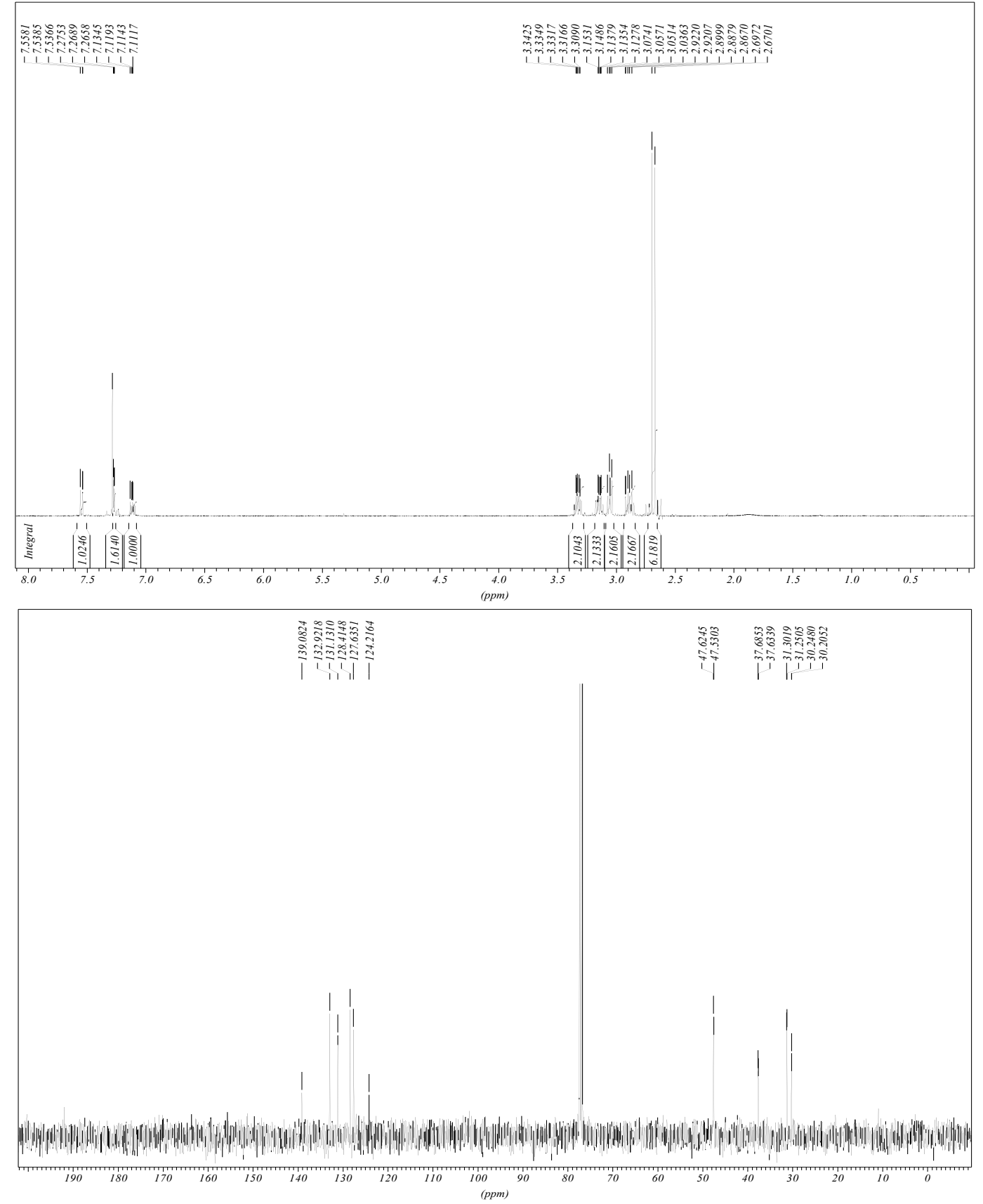


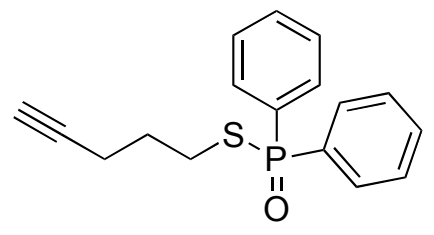

5a: Following GP1, substrate 5a was isolated (petroleum ether/ethyl acetate 1:1, $978 \mathrm{mg}, 56 \%$ ) as a whitish dense oil. IR (neat): 3295, 3056, 2926, 2117, 1588, $1194 \mathrm{~cm}^{-1} ;{ }^{1} \mathrm{H} \mathrm{NMR}\left(\mathrm{CDCl}_{3}, 400 \mathrm{MHz}\right)$ : $\delta 1.85$ (quint., $J=7.1 \mathrm{~Hz}, 2 \mathrm{H}, \mathrm{SCH}_{2} \mathrm{CH}_{2}$ ), 1.90 (t, $\left.J=2.7 \mathrm{~Hz}, 1 \mathrm{H}, \equiv \mathrm{CH}\right), 2.25$ (td, $J=7.1,2.7 \mathrm{~Hz}$, $\left.2 \mathrm{H}, \equiv \mathrm{CCH}_{2}\right), 2.91\left(\mathrm{td}, J=11.4,7.1 \mathrm{~Hz}, 2 \mathrm{H}, \mathrm{SCH}_{2}\right), 7.43-7.56(\mathrm{~m}, 6 \mathrm{H}$, arom.), 7.84-7.91 (m, $4 \mathrm{H}$, arom.); ${ }^{13} \mathrm{C} \mathrm{NMR}\left(\mathrm{CDCl}_{3}, 100 \mathrm{MHz}\right): \delta 17.3\left(\mathrm{SCH}_{2} \mathrm{CH}_{2}\right), 28.2\left(\equiv \mathrm{CCH}_{2}\right), 29.2\left(\mathrm{~d}, J=4.3 \mathrm{~Hz}, \mathrm{SCH}_{2}\right)$, $69.3(\equiv \mathrm{CH}), 82.7\left(\equiv C \mathrm{CH}_{2}\right), 128.7(\mathrm{~d}, J=12.8 \mathrm{~Hz}, \mathrm{CH}$ arom.), 131.5 (d, $J=10.3 \mathrm{~Hz}, \mathrm{CH}$ arom.), 132.4 (d, $J=3.4 \mathrm{~Hz}, \mathrm{CH}$ arom.), 133.2 (d, $J=106.3 \mathrm{~Hz}, \mathrm{C}$ arom.); ${ }^{31} \mathrm{P}$ NMR $\left(\mathrm{CDCl}_{3}, 162 \mathrm{MHz}\right): \delta$ 44.6.
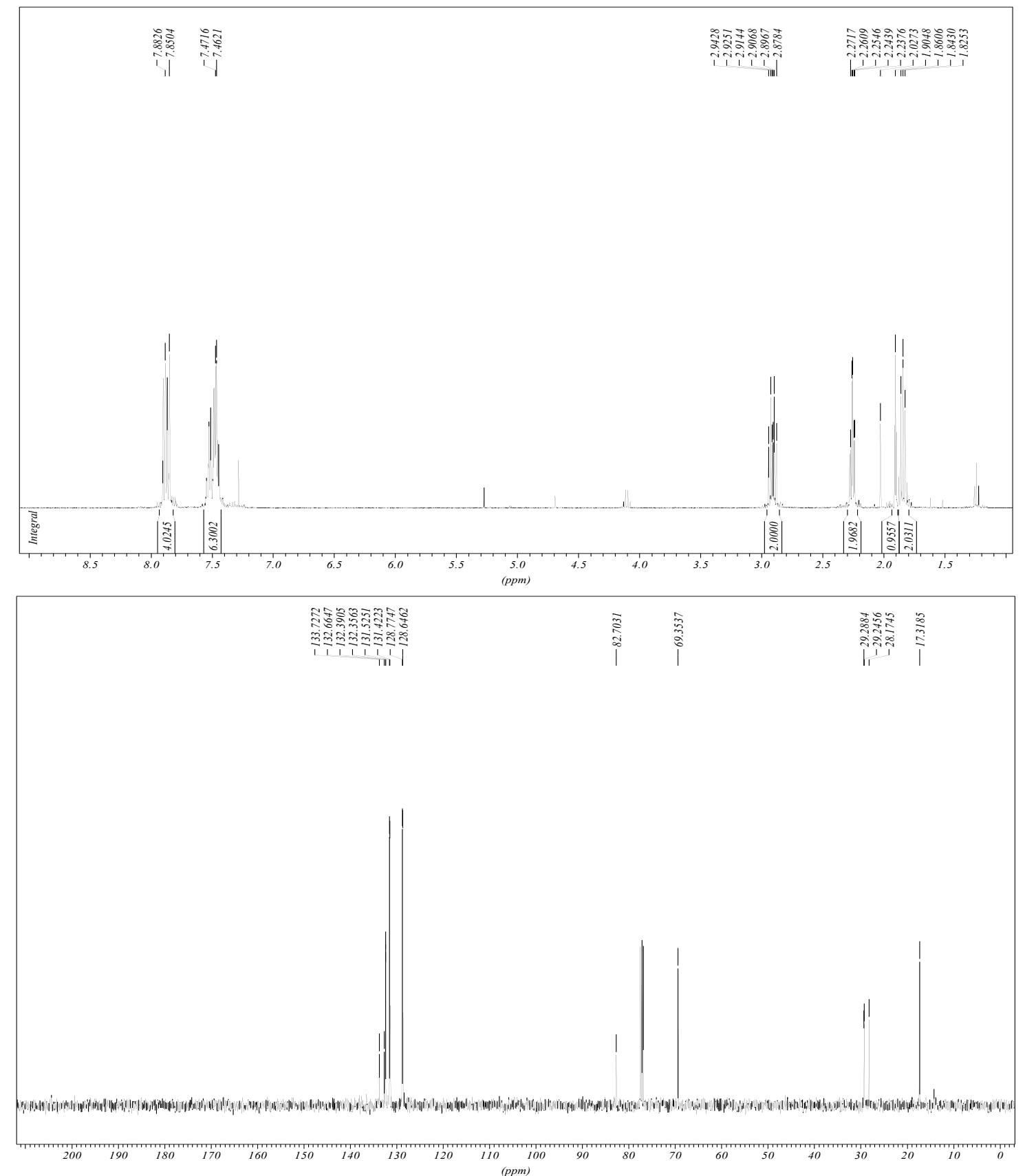


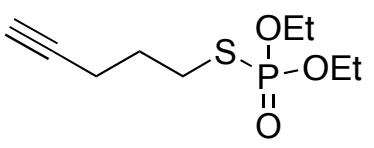

5b: Following GP1, substrate 5b was isolated (petroleum ether/ethyl acetate 1:1, $602 \mathrm{mg}, 51 \%$ ) as a yellow oil. IR (neat): 3232, 2982, 2117, $1162 \mathrm{~cm}^{-1} ;{ }^{1} \mathrm{H} \mathrm{NMR}\left(\mathrm{CDCl}_{3}, 400 \mathrm{MHz}\right): \delta 1.33$ (t, $J=7.1 \mathrm{~Hz}, 6 \mathrm{H}, \mathrm{OCH}_{2} \mathrm{Me}$ ), 1.90 (quint., $J=7.1 \mathrm{~Hz}, 2 \mathrm{H}, \mathrm{SCH}_{2} \mathrm{CH}_{2}$ ), 1.96 (t, $J=2.7 \mathrm{~Hz}, 1 \mathrm{H}, \equiv \mathrm{CH}$ ), $2.31\left(\mathrm{td}, J=7.1,2.7 \mathrm{~Hz}, 2 \mathrm{H}, \equiv \mathrm{CCH}_{2}\right), 2.93\left(\mathrm{td}, J=11.4,7.1 \mathrm{~Hz}, 2 \mathrm{H}, \mathrm{SCH}_{2}\right), 4.05-4.22(\mathrm{~m}, 4 \mathrm{H}$, $\left.\mathrm{OCH}_{2}\right) ;{ }^{13} \mathrm{C} \mathrm{NMR}\left(\mathrm{CDCl}_{3}, 100 \mathrm{MHz}\right): \delta 16.1\left(\mathrm{~d}, J=6.9 \mathrm{~Hz}, \mathrm{OCH}_{2} \mathrm{Me}\right), 17.3\left(\mathrm{SCH}_{2} \mathrm{CH}_{2}\right), 29.4(\mathrm{~d}$, $J=4.8 \mathrm{~Hz}, \mathrm{SCH}_{2}$ or $\left.\equiv \mathrm{CCH}_{2}\right), 29.6\left(\mathrm{~d}, J=4.3 \mathrm{~Hz}, \mathrm{SCH}_{2}\right.$ or $\left.\equiv \mathrm{CCH}_{2}\right), 63.5\left(\mathrm{~d}, J=6.0 \mathrm{~Hz}, \mathrm{OCH}_{2}\right), 69.3$ $(\equiv \mathrm{CH}), 82.7\left(\equiv \mathrm{CCH}_{2}\right) ;{ }^{31} \mathrm{P} \mathrm{NMR}\left(\mathrm{CDCl}_{3}, 162 \mathrm{MHz}\right): \delta 29.0$.

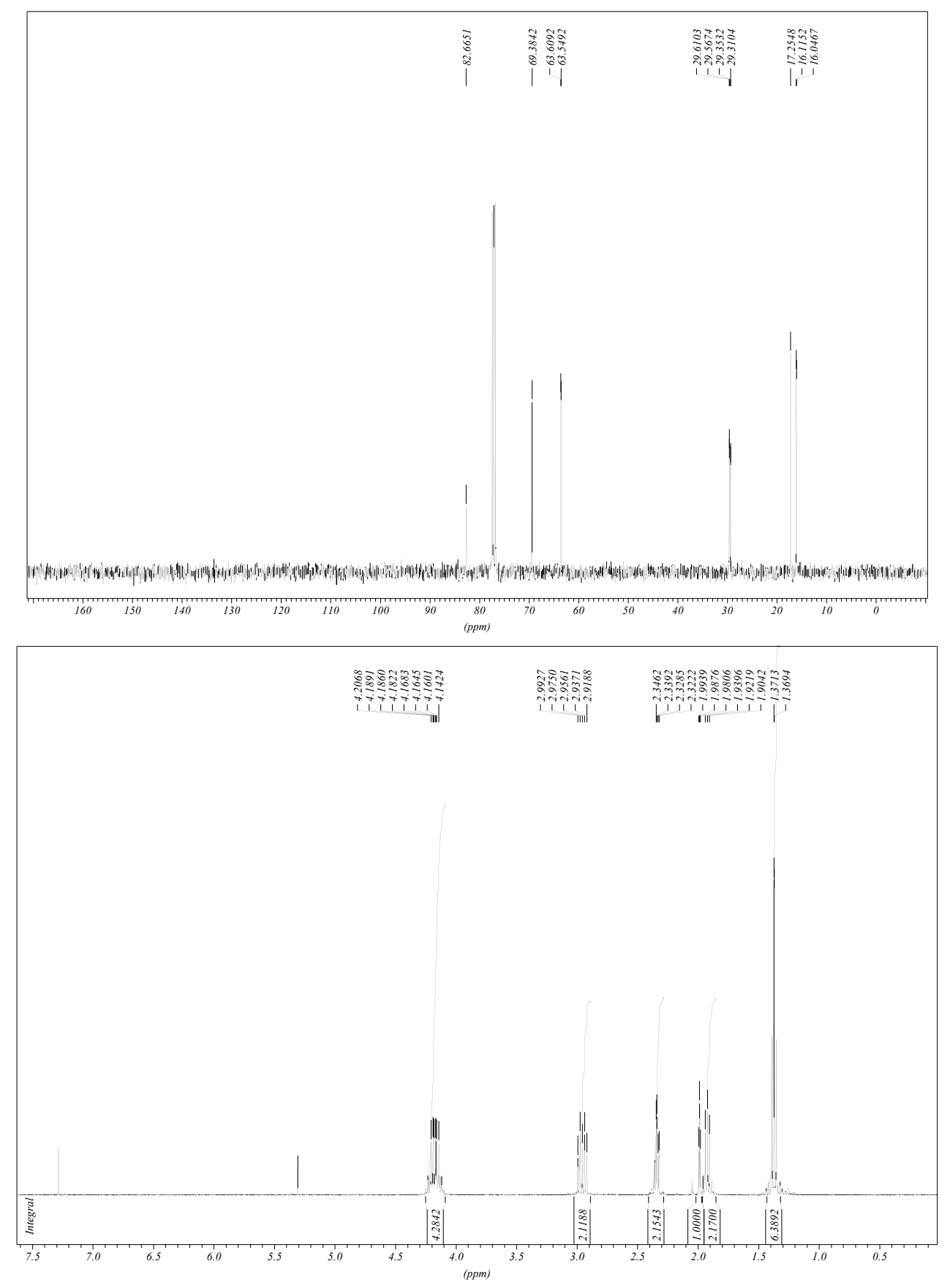




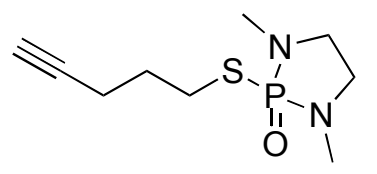

5c: Following GP1, substrate 5c was isolated $\left(\mathrm{CH}_{2} \mathrm{Cl}_{2} / \mathrm{MeOH} 9: 1,488 \mathrm{mg}, 42 \%\right)$ as a yellow oil. IR (neat): 3213, 2928, 1475, $1160 \mathrm{~cm}^{-1}$; ${ }^{1} \mathrm{H} \mathrm{NMR}\left(\mathrm{CDCl}_{3}, 400 \mathrm{MHz}\right): \delta 1.83$ (quint., $J=7.1 \mathrm{~Hz}, 2 \mathrm{H}$, $\left.\mathrm{SCH}_{2} \mathrm{CH}_{2}\right), 1.96(\mathrm{t}, J=2.7 \mathrm{~Hz}, 1 \mathrm{H}, \equiv \mathrm{CH}), 2.31\left(\mathrm{td}, J=7.1,2.7 \mathrm{~Hz}, 2 \mathrm{H}, \equiv \mathrm{CCH}_{2}\right), 2.68(\mathrm{~s}, 3 \mathrm{H}$, $\mathrm{NMe}$ ), 2.71 (s, $3 \mathrm{H}, \mathrm{NMe}), 2.73$ (td, $\left.J=11.4,7.1 \mathrm{~Hz}, 2 \mathrm{H}, \mathrm{SCH}_{2}\right), 3.05-3.14\left(\mathrm{~m}, 2 \mathrm{H}, \mathrm{NCH}_{2}\right), 3.27-$ $3.37\left(\mathrm{~m}, 2 \mathrm{H}, \mathrm{NCH}_{2}\right) ;{ }^{13} \mathrm{C} \mathrm{NMR}\left(\mathrm{CDCl}_{3}, 100 \mathrm{MHz}\right): \delta 17.4\left(\mathrm{SCH}_{2} \mathrm{CH}_{2}\right), 29.5\left(\mathrm{~d}, J=5.1 \mathrm{~Hz}, \mathrm{SCH}_{2}\right.$ or $\left.\equiv \mathrm{CCH}_{2}\right), 29.6\left(\mathrm{~d}, J=4.3 \mathrm{~Hz}, \mathrm{SCH}_{2}\right.$ or $\left.\equiv \mathrm{CCH}_{2}\right), 31.3\left(\mathrm{~d}, J=6.0 \mathrm{~Hz}, \mathrm{NCH}_{2}\right), 47.5(\mathrm{~d}, J=10.3 \mathrm{~Hz}$, $\mathrm{NMe}), 69.1$ ( $\equiv \mathrm{CH}), 83.1\left(\equiv \mathrm{CCH}_{2}\right) ;{ }^{31} \mathrm{P} \mathrm{NMR}\left(\mathrm{CDCl}_{3}, 162 \mathrm{MHz}\right): \delta 42.0$.
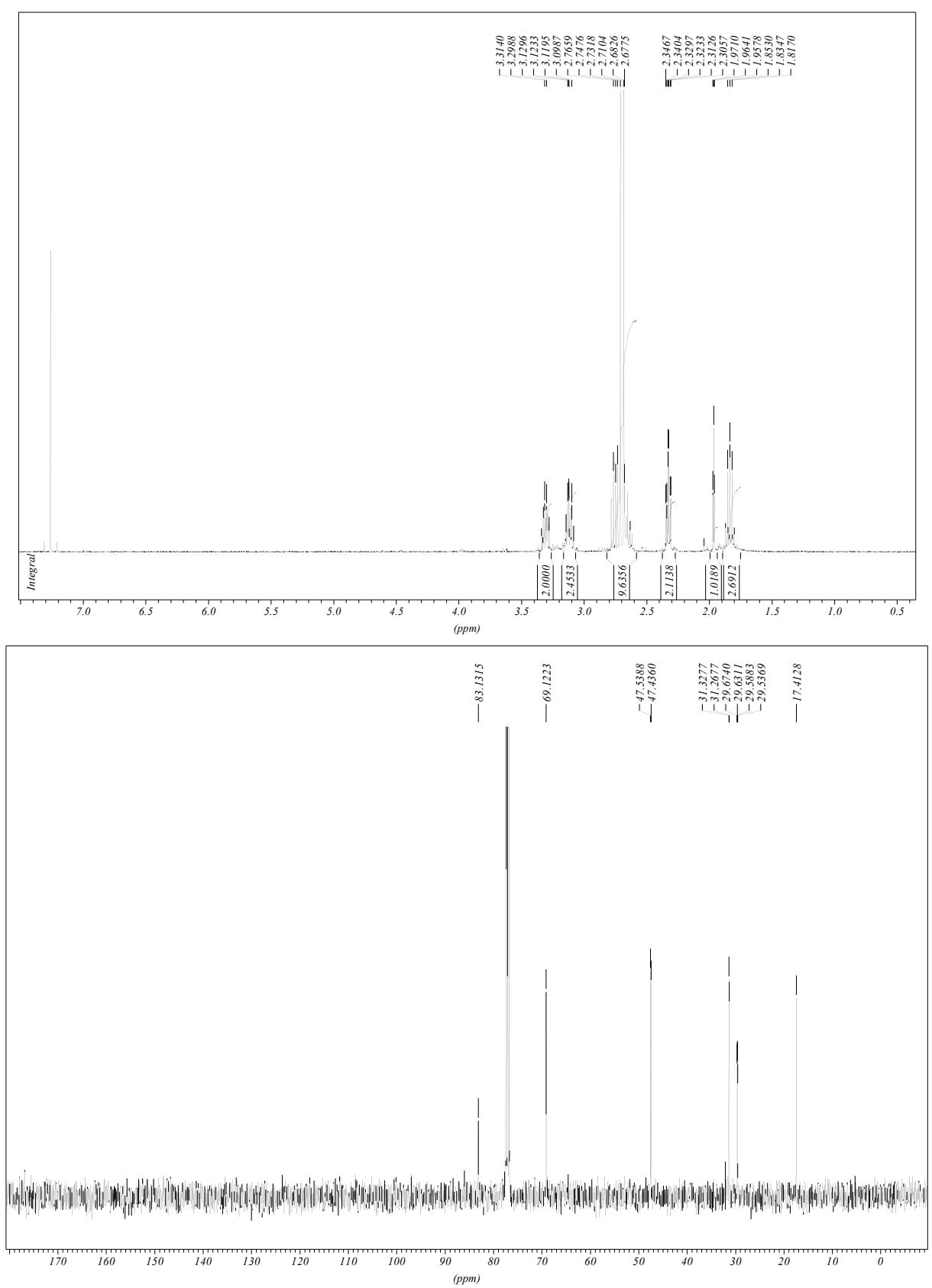


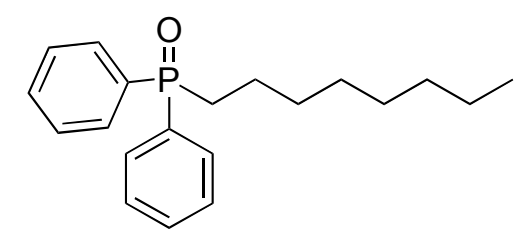

2a: Following GP2, substrate 2a was isolated (petroleum ether/ethyl acetate 50:50, $132 \mathrm{mg}, 84 \%$ ) as a white solid. M. p. $57-58.5^{\circ} \mathrm{C}$; IR (neat): $3055,2924,2853,1181,1119 \mathrm{~cm}^{-1} ;{ }^{1} \mathrm{H} \mathrm{NMR}\left(\mathrm{CDCl}_{3}\right.$, $400 \mathrm{MHz}): \delta 0.86(\mathrm{t}, J=7.1 \mathrm{~Hz}, 3 \mathrm{H}, \mathrm{Me}), 1.18-1.31\left(\mathrm{~m}, 8 \mathrm{H}, \mathrm{CH}_{2}\right), 1.35-1.44\left(\mathrm{~m}, 2 \mathrm{H}, \mathrm{CH}_{2}\right), 1.57-$ $1.66\left(\mathrm{~m}, 2 \mathrm{H}, \mathrm{CH}_{2}\right), 2.22-2.30\left(\mathrm{~m}, 2 \mathrm{H}, \mathrm{CH}_{2}\right), 7.44-7.53$ (m, $6 \mathrm{H}$ arom.), 7.71-7.78 (m, $4 \mathrm{H}$ arom.);

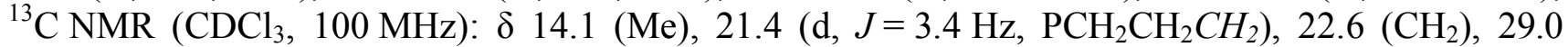
$\left(\mathrm{CH}_{2}\right), 29.7\left(\mathrm{~d}, J=71.1 \mathrm{~Hz}, \mathrm{PCH}_{2}\right), 31.0$ (d, $\left.J=14.6 \mathrm{~Hz}, \mathrm{PCH}_{2} \mathrm{CH}_{2}\right), 31.8\left(\mathrm{CH}_{2}\right), 128.6$ (d, $J=12.0 \mathrm{~Hz}, \mathrm{CH}$ arom.), 130.8 (d, $J=9.4 \mathrm{~Hz}, \mathrm{CH}$ arom.), 131.6 (d, $J=3.4 \mathrm{~Hz}, \mathrm{CH}$ arom.), 133.2 (d, $J=96.8 \mathrm{~Hz}, \mathrm{C}$ arom.); ${ }^{31} \mathrm{P} \mathrm{NMR}\left(\mathrm{CDCl}_{3}, 162 \mathrm{MHz}\right): \delta 34.1$; HRMS calcd. for $\mathrm{C}_{20} \mathrm{H}_{28} \mathrm{OP}\left(\mathrm{MH}^{+}\right)$: 315.1878 , found 315.1878 .
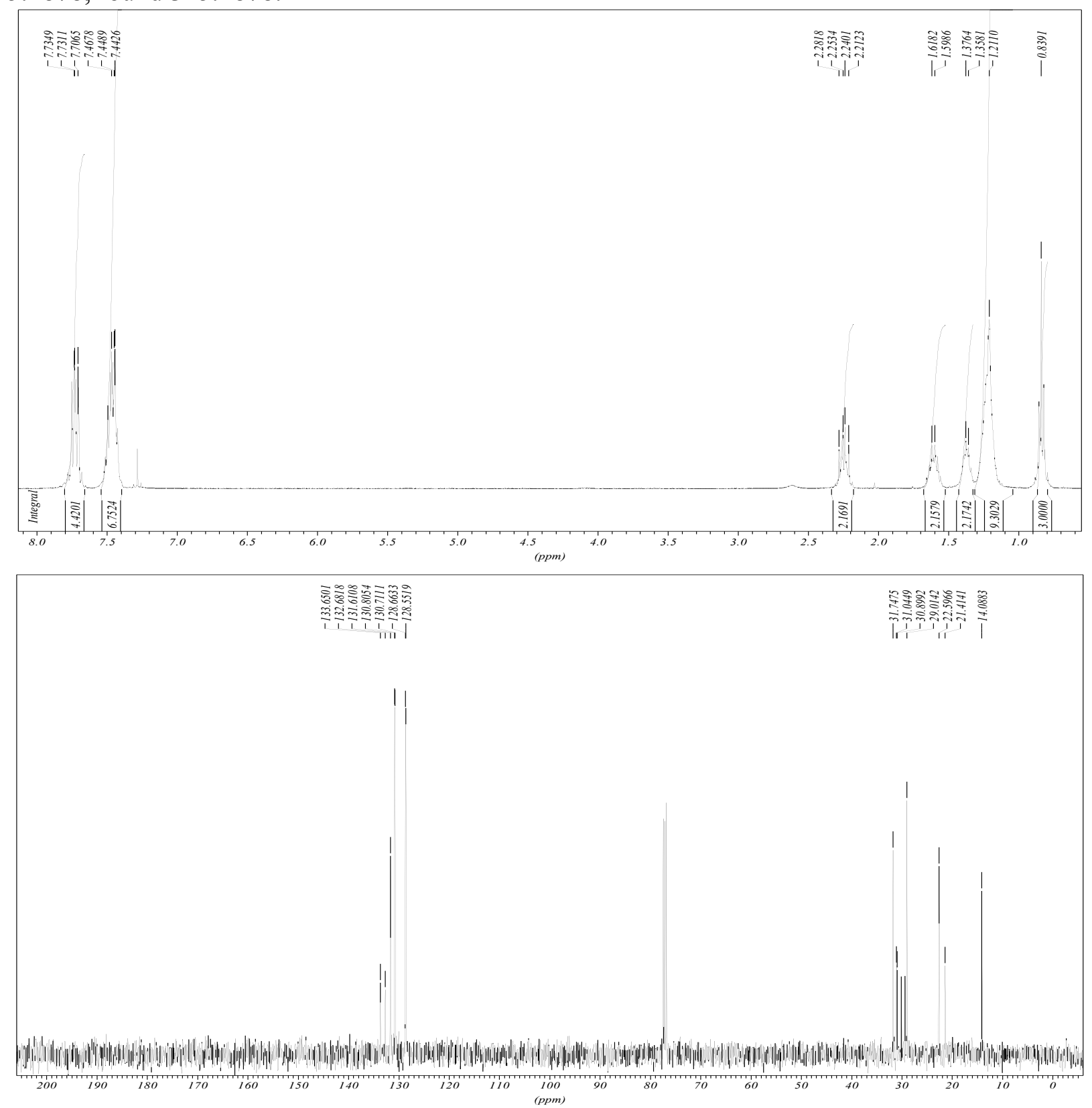


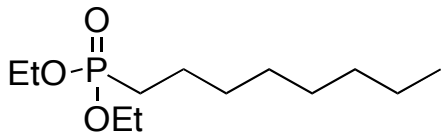

2b: Following GP2, substrate $\mathbf{2 b}$ was isolated $\left(\mathrm{CH}_{2} \mathrm{Cl}_{2} /\right.$ ethyl acetate 50:50, $\left.94 \mathrm{mg}, 75 \%\right)$ as a colorless oil. Its spectral data correspond to those of commercial samples (CAS number: 1068-07-1).
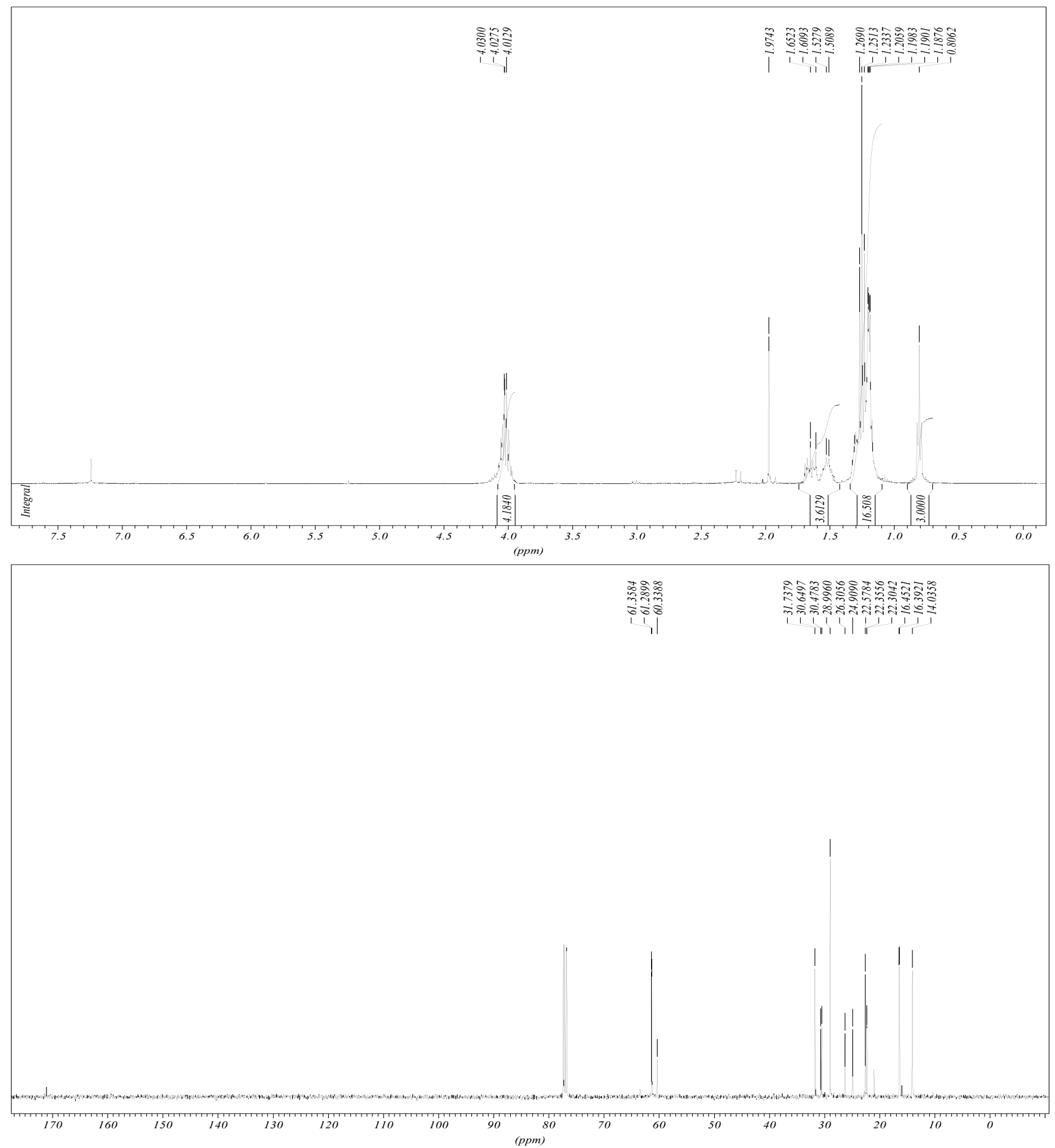


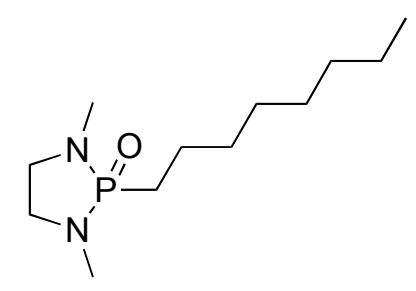

2c: Following GP2, substrate $2 c$ was isolated $\left(\mathrm{CH}_{2} \mathrm{Cl}_{2} /\right.$ methanol 90:10, $\left.74 \mathrm{mg}, 60 \%\right)$ as a colorless oil. IR (neat): 2923, 2853, $1156 \mathrm{~cm}^{-1} ;{ }^{1} \mathrm{H} \mathrm{NMR}\left(\mathrm{CDCl}_{3}, 400 \mathrm{MHz}\right): \delta 0.88$ (t, $\left.J=7.1 \mathrm{~Hz}, 3 \mathrm{H}, \mathrm{Me}\right)$, 1.18-1.37 (m, $\left.12 \mathrm{H}, \mathrm{CH}_{2}\right), 1.76-1.84\left(\mathrm{~m}, 2 \mathrm{H}, \mathrm{CH}_{2} \mathrm{P}\right), 2.66$ (d, $\left.J=9.4 \mathrm{~Hz}, 6 \mathrm{H}, \mathrm{NMe}\right), 3.04-3.08$ (m, $\left.2 \mathrm{H}, \mathrm{NCH}_{2}\right), 3.16-3.23\left(\mathrm{~m}, 2 \mathrm{H}, \mathrm{NCH}_{2}\right) ;{ }^{13} \mathrm{C} \mathrm{NMR}\left(\mathrm{CDCl}_{3}, 100 \mathrm{MHz}\right): \delta 14.1\left(\mathrm{CH}_{3}\right), 22.6\left(\mathrm{CH}_{2}\right), 23.0$ (d, $\left.J=4.3 \mathrm{~Hz}, \mathrm{CH}_{2}\right), 26.9$ (d, $\left.J=115.7 \mathrm{~Hz}, \mathrm{PCH}_{2}\right), 29.0\left(\mathrm{CH}_{2}\right), 29.1\left(\mathrm{CH}_{2}\right), 30.7$ (d, $J=17.1 \mathrm{~Hz}$, $\left.\mathrm{CH}_{2}\right), 31.7\left(\mathrm{CH}_{2}\right), 31.9$ (d, $\left.J=5.1 \mathrm{~Hz}, \mathrm{NMe}\right), 48.3\left(\mathrm{NCH}_{2}\right) ;{ }^{31} \mathrm{P} \mathrm{NMR}\left(\mathrm{CDCl}_{3}, 162 \mathrm{MHz}\right): \delta$ 43.3; HRMS calcd. for $\mathrm{C}_{12} \mathrm{H}_{28} \mathrm{~N}_{2} \mathrm{OP}\left([\mathrm{M}+\mathrm{H}]^{+}\right): 247.1939$, found 247.1934 .
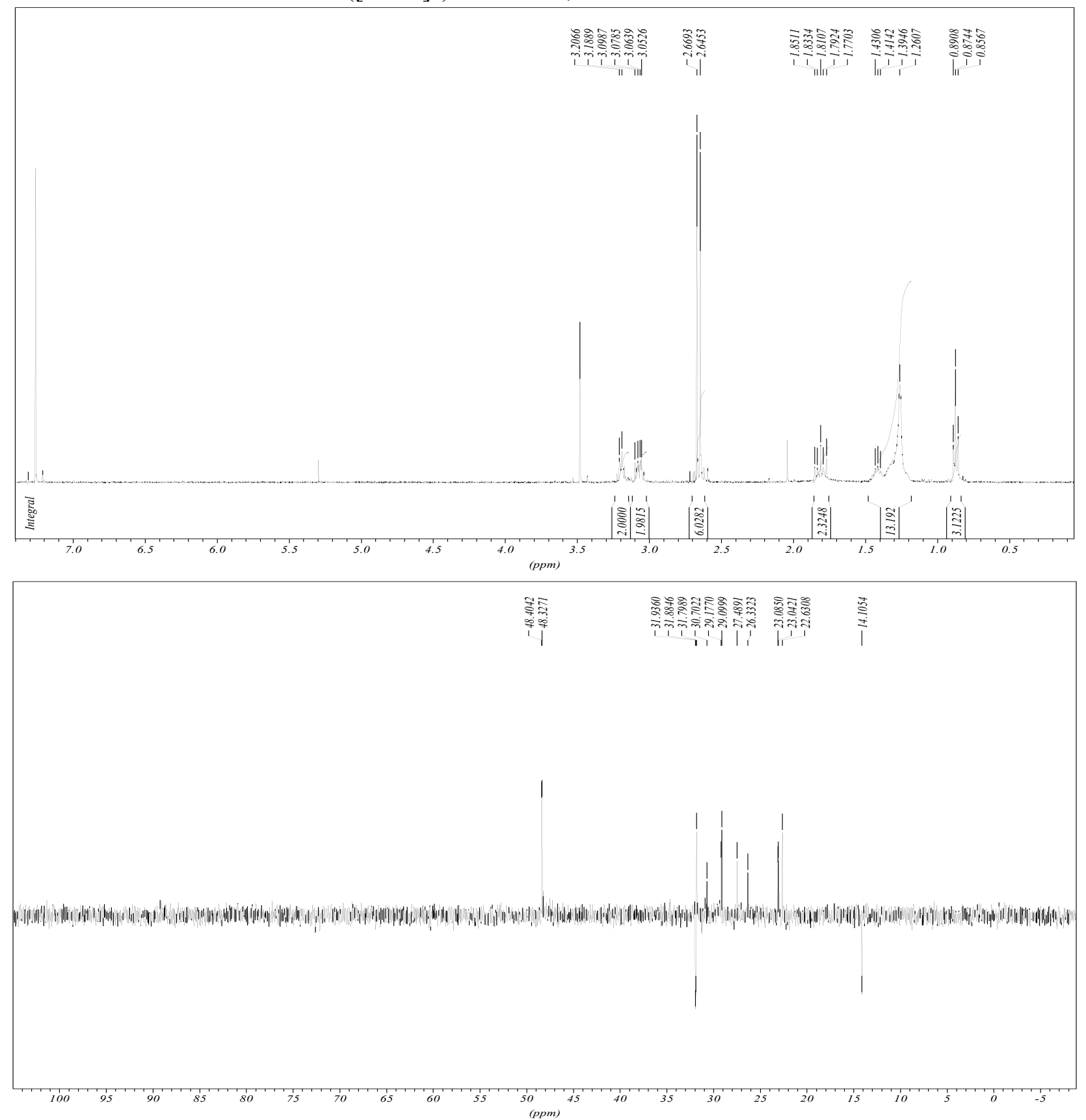


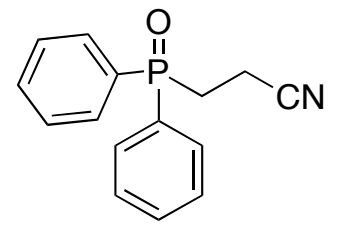

3a: Following GP3, substrate 3a was isolated (petroleum ether/ethyl acetate 50:50, $64 \mathrm{mg}, 50 \%$ ) as an oil. Spectral data corresponded to those reported in the literature.
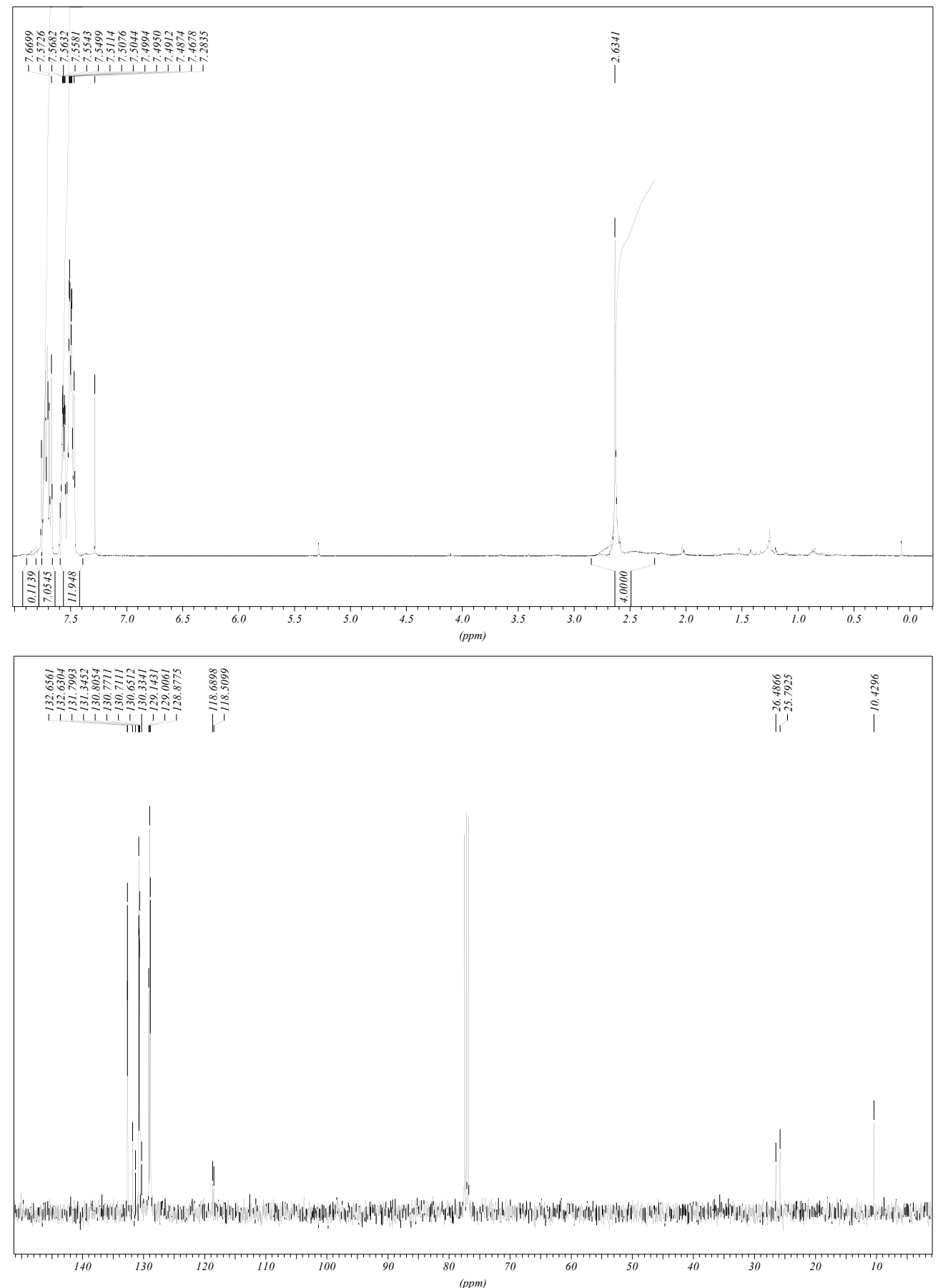

${ }^{1}$ (a) Hall, C. D.; Lowther, N.; Tweedy, B. R.; Hall, A. C.; Shaw, G. J. Chem. Soc., Perkin Trans. 2 1998, 2047; (b) Stockland, R. A., Jr.; Taylor, R. I.; Thompson, L. E.; Patel, P. B. Org. Lett. 2005, 7, 851. 
<smiles>CCOP(=O)(CCC#N)OCC</smiles>

3b: Following GP2, substrate $\mathbf{3 b}$ was isolated (ethyl acetate, $71 \mathrm{mg}, 74 \%$ ) as a colorless oil. Its spectal data correspond to those of commercial samples (CAS number: 10123-62-3).

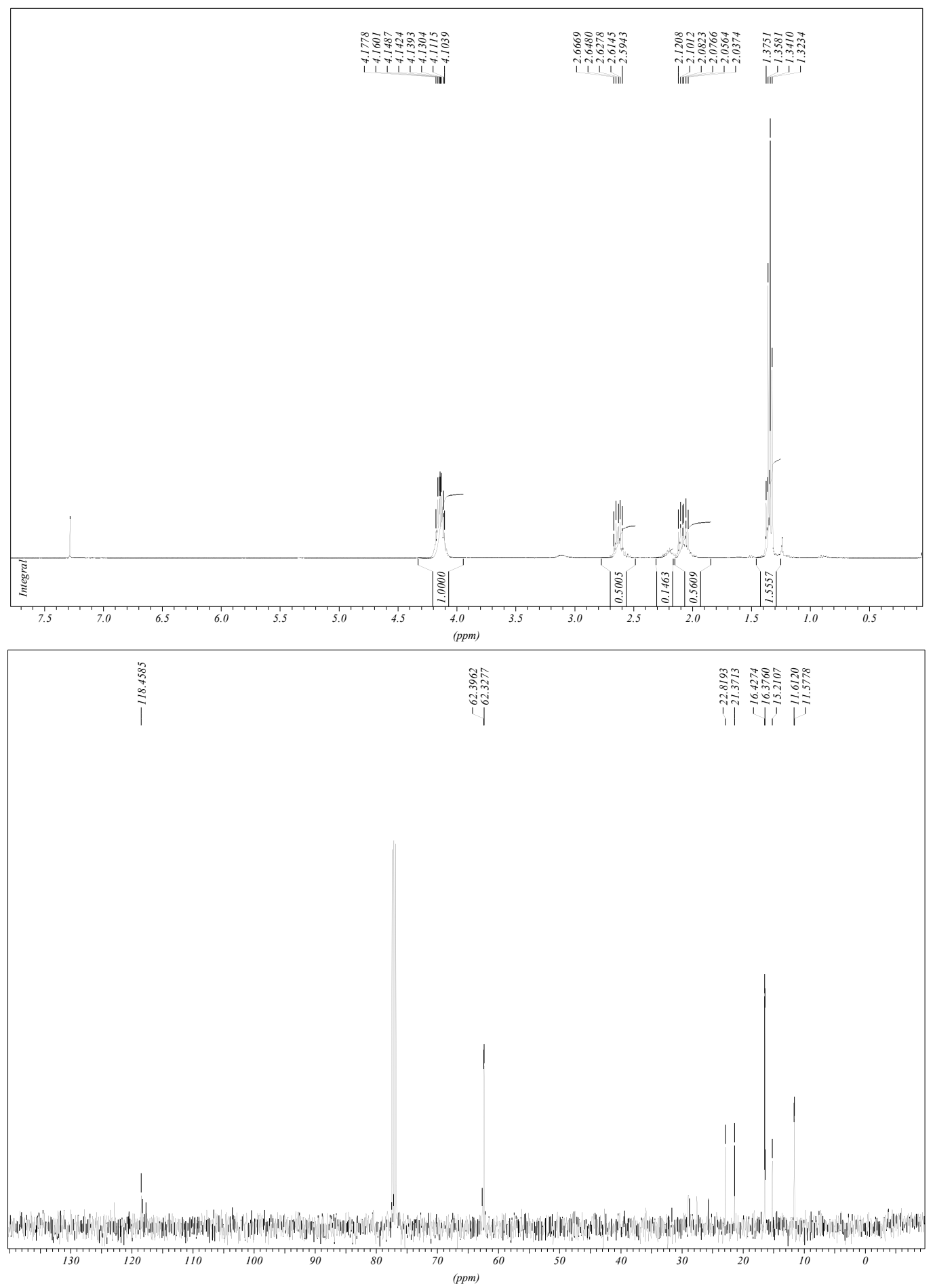




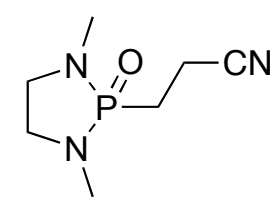

3c: Following GP2, substrate 3c was isolated $\left(\mathrm{CH}_{2} \mathrm{Cl}_{2} /\right.$ methanol 90:10, $\left.77 \mathrm{mg}, 82 \%\right)$ as a white solid. M. p. $112-115^{\circ} \mathrm{C}$; IR (neat): 2912, 2866, 2361, $1168 \mathrm{~cm}^{-1} ;{ }^{1} \mathrm{H} \mathrm{NMR}\left(\mathrm{CDCl}_{3}, 400 \mathrm{MHz}\right): \delta 2.16$ (dt, $\left.J=15.4,7.8 \mathrm{~Hz}, 2 \mathrm{H}, \mathrm{CH}_{2}\right), 2.47$ (dt, $\left.J=15.6,7.8 \mathrm{~Hz}, 2 \mathrm{H}, \mathrm{CH}_{2}\right), 2.67$ (d, $J=9.6 \mathrm{~Hz}, 6 \mathrm{H}, \mathrm{NMe}$ ), 3.12-3.26 (m, $\left.4 \mathrm{H}, \mathrm{NCH}_{2}\right) ;{ }^{13} \mathrm{C} \mathrm{NMR}\left(\mathrm{CDCl}_{3}, 100 \mathrm{MHz}\right): \delta 12.3\left(\mathrm{~d}, J=4.3 \mathrm{~Hz}, \mathrm{CH}_{2} \mathrm{CN}\right), 22.4(\mathrm{~d}$, $\left.J=120.0 \mathrm{~Hz}, \mathrm{CH}_{2} \mathrm{P}\right), 31.6(\mathrm{~d}, J=5.1 \mathrm{~Hz}, \mathrm{NMe}), 47.9\left(\mathrm{~d}, J=9.4 \mathrm{~Hz}, \mathrm{NCH}_{2}\right), 119.0(\mathrm{CN}) ;{ }^{31} \mathrm{P} \mathrm{NMR}$ $\left(\mathrm{CDCl}_{3}, 162 \mathrm{MHz}\right): \delta 37.4$; HRMS calcd. for $\mathrm{C}_{7} \mathrm{H}_{14} \mathrm{~N}_{3} \mathrm{OPNa}\left([\mathrm{M}+\mathrm{Na}]^{+}\right): 210.0772$, found 210.0782 .
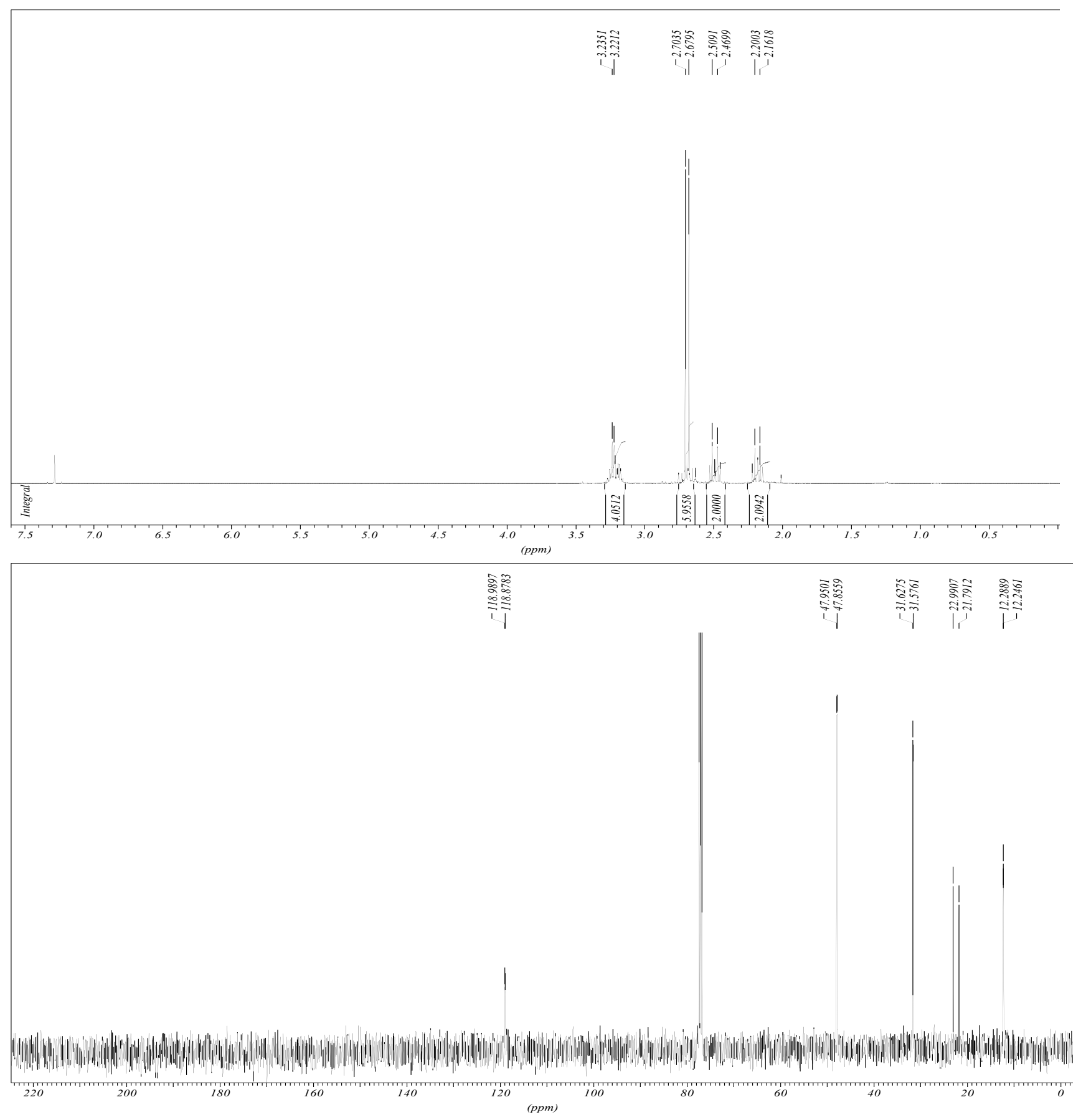


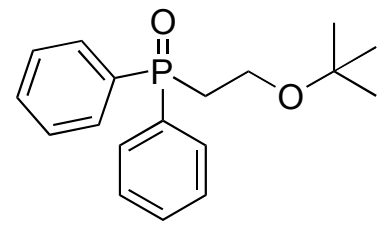

4a: Following GP2, substrate 4a was isolated (petroleum ether/ethyl acetate 50:50, $107 \mathrm{mg}, 71 \%$ ) as an unseparable mixture of compounds (80-90\% pure). IR (neat): $3055,2972,1187,1118 \mathrm{~cm}^{-1}$; ${ }^{1} \mathrm{H}$ NMR $\left(\mathrm{CDCl}_{3}, 400 \mathrm{MHz}\right): \delta 1.07\left(\mathrm{~s}, 9 \mathrm{H}, \mathrm{CMe}_{3}\right), 2.53-2.66\left(\mathrm{~m}, 2 \mathrm{H}, \mathrm{CH}_{2} \mathrm{P}\right), 3.66-3.78(\mathrm{~m}, 2 \mathrm{H}$, $\mathrm{CH}_{2} \mathrm{O}$ ), 7.45-7.55 (m, $6 \mathrm{H}$ arom.), 7.70-7.78 (m, $4 \mathrm{H}$ arom.); ${ }^{13} \mathrm{C} \mathrm{NMR}\left(\mathrm{CDCl}_{3}, 50 \mathrm{MHz}\right): \delta 27.7$ $\left(\mathrm{CMe}_{3}\right), 32.0\left(\mathrm{~d}, J=70.6 \mathrm{~Hz}, \mathrm{PCH}_{2}\right), 55.6\left(\mathrm{CH}_{2} \mathrm{O}\right), 73.9\left(\mathrm{CMe}_{3}\right), 128.9$ (d, $J=11.9 \mathrm{~Hz}, \mathrm{CH}$ arom.), 131.1 (d, $J=9.6 \mathrm{~Hz}, \mathrm{CH}$ arom.), 132.0 (d, $J=2.9 \mathrm{~Hz}, \mathrm{CH}$ arom.), 133.7 (d, $J=99.1 \mathrm{~Hz}, \mathrm{C}$ arom.);

${ }^{31} \mathrm{P}$ NMR $\left(\mathrm{CDCl}_{3}, 162 \mathrm{MHz}\right): \delta 33.2$.

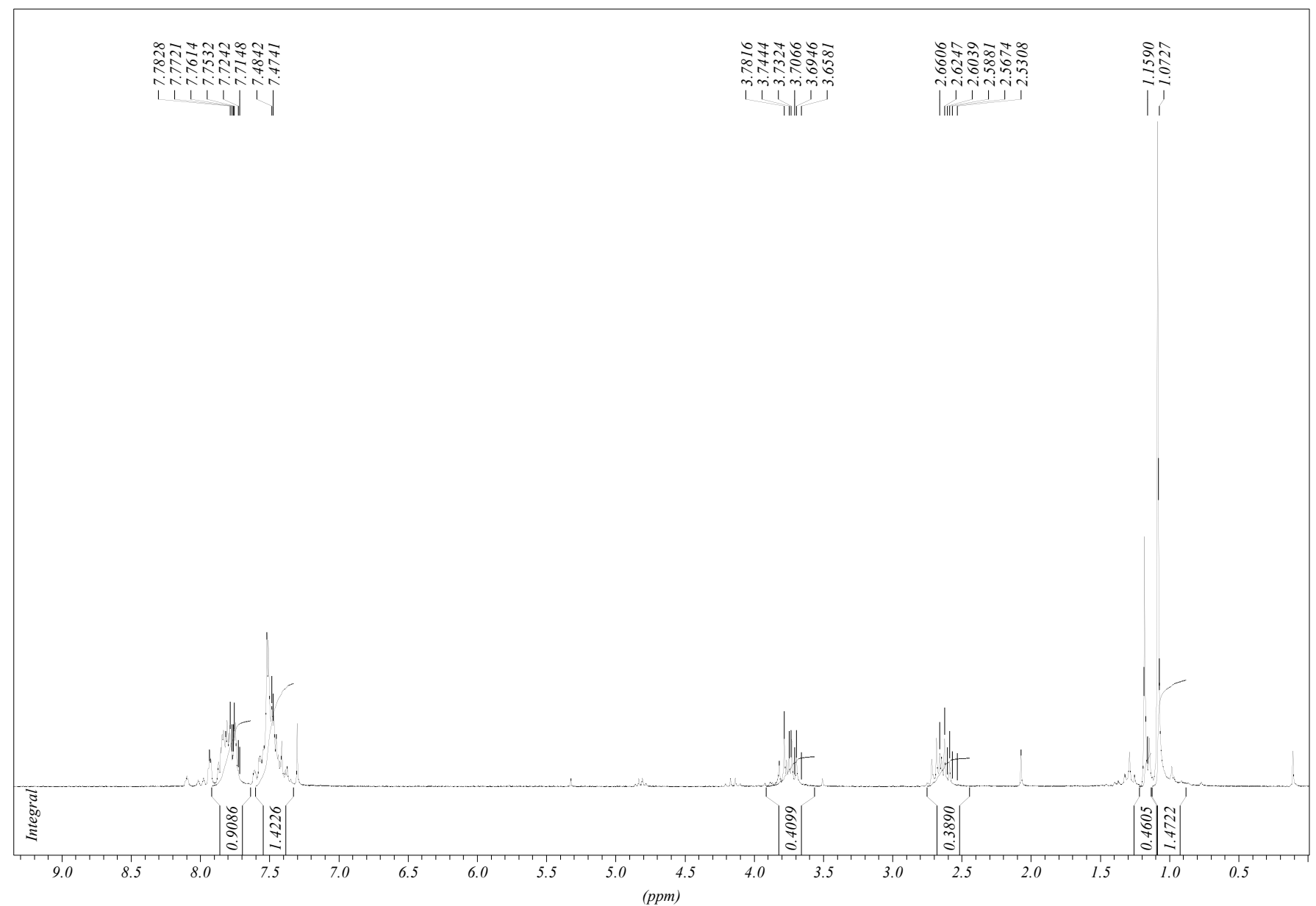


<smiles>CCOP(=O)(CC)OCC(C)(C)C</smiles>

4b: Following GP2, substrate 4b was isolated $\left(\mathrm{CH}_{2} \mathrm{Cl}_{2} / \mathrm{MeOH} 90: 10,67 \mathrm{mg}, 56 \%\right)$ as an oil. Spectral data corresponded to those reported in the literature. ${ }^{2}$
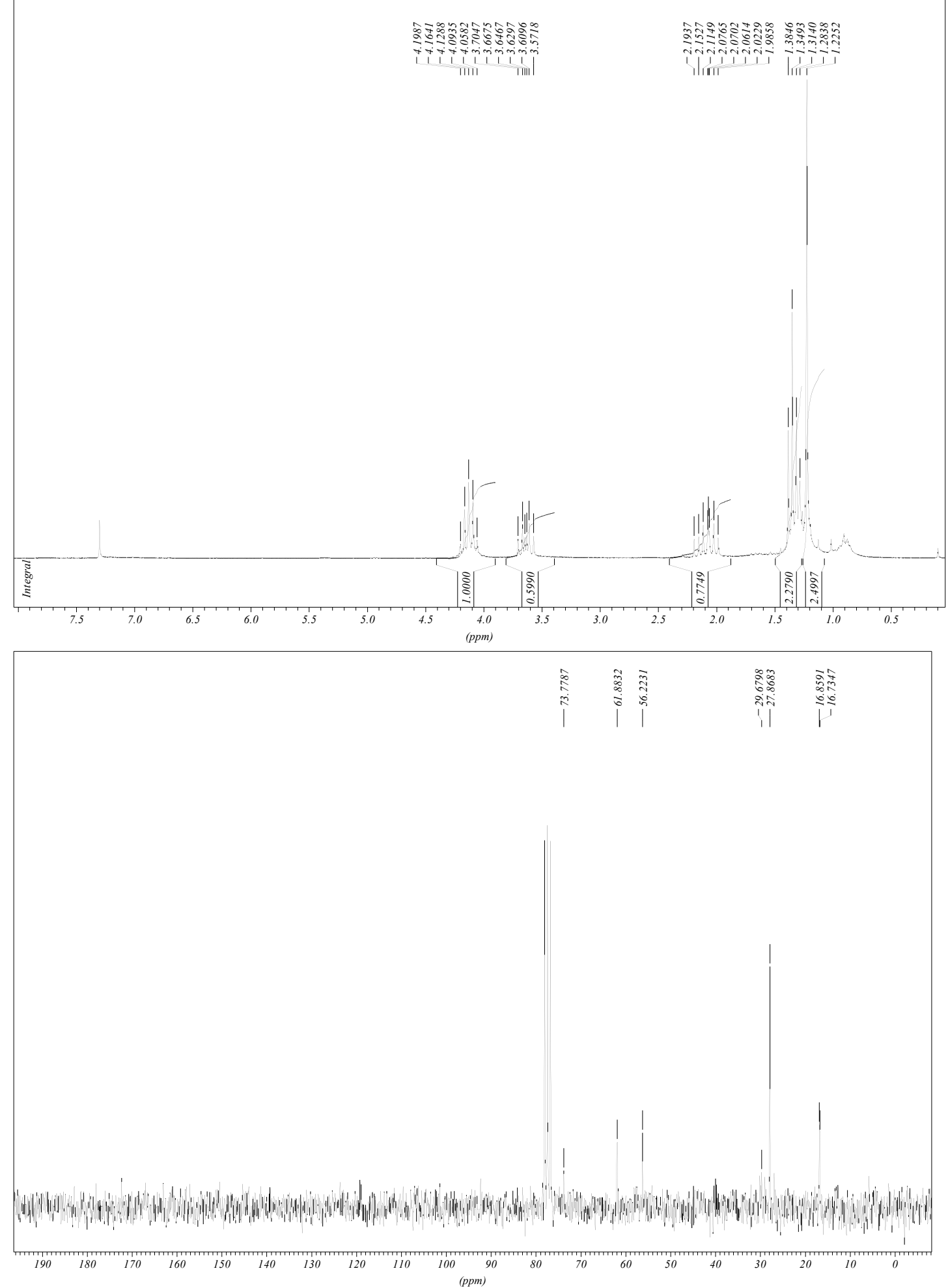

${ }^{2}$ Griffin, C. E.; Kundu, S. K. J. Org. Chem. 1969, 34, 1532. 


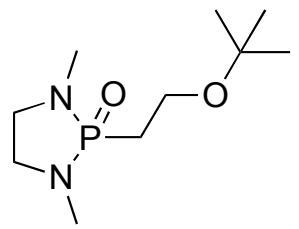

4c: Following GP2, substrate 4c was isolated (ethyl acetate/methanol 90:10, $47 \mathrm{mg}, 40 \%$ ) as an unstable oil. ${ }^{1} \mathrm{H}$ NMR (MeOD, $\left.200 \mathrm{MHz}\right): \delta 1.23\left(\mathrm{~s}, 9 \mathrm{H}, \mathrm{CMe}_{3}\right), 2.13$ (dt, $J=15.6,6.4 \mathrm{~Hz}, 2 \mathrm{H}$, $\left.\mathrm{CH}_{2} \mathrm{P}\right), 2.68(\mathrm{~d}, J=9.8 \mathrm{~Hz}, 6 \mathrm{H}, \mathrm{NMe}), 3.16-3.26\left(\mathrm{~m}, 4 \mathrm{H}, \mathrm{CH}_{2} \mathrm{~N}\right), 3.56(\mathrm{dt}, J=18.6,6.4 \mathrm{~Hz}, 2 \mathrm{H}$, $\mathrm{CH}_{2} \mathrm{O} ;{ }^{31} \mathrm{P}$ NMR $\left(\mathrm{CDCl}_{3}, 162 \mathrm{MHz}\right): \delta 40.5$; ES-MS $\left(\mathrm{MeOH} / \mathrm{CH}_{2} \mathrm{Cl}_{2}\right): 257.2\left([\mathrm{M}+\mathrm{Na}]^{+}, 100\right), 235.2$ $\left(\mathrm{MH}^{+}, 22\right)$.

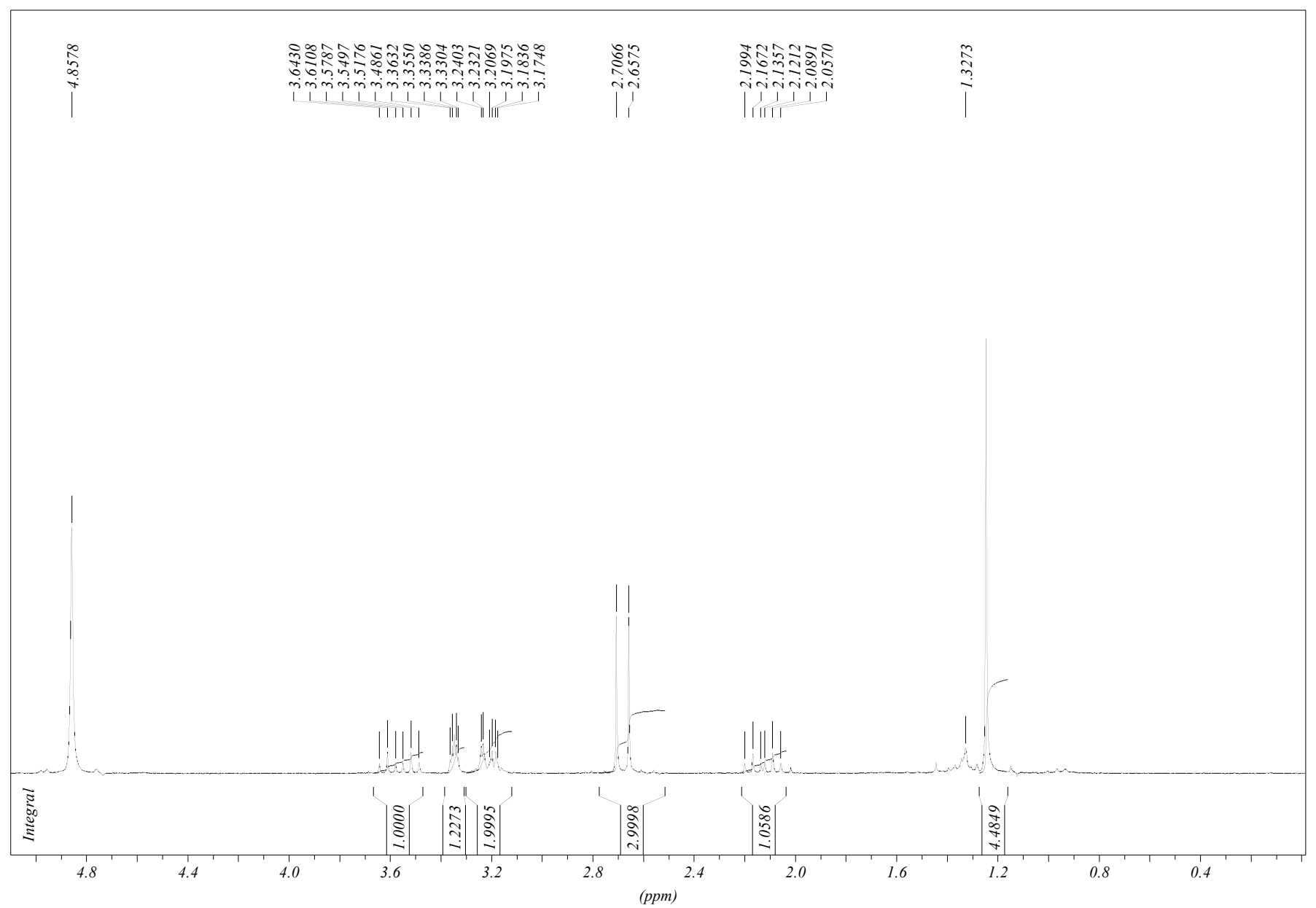




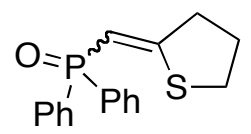

To a degassed, refluxed solution of $\mathbf{5 a}(0.5 \mathrm{mmol}$; 1 equiv.; $150 \mathrm{mg})$ in toluene $(6 \mathrm{ml})$ was added a solution of AIBN ( $0.1 \mathrm{mmol}$; 0.2 equiv.; $15 \mathrm{mg})$ and $\mathrm{PhSH}(0.2 \mathrm{mmol}, 0.4$ equiv., $21 \mu \mathrm{L})$ in toluene ( $5 \mathrm{mmol})$ via syringe pump $\left(0.03 \mathrm{mmol} . \mathrm{h}^{-1}\right)$. After completion of the addition, the reaction was refluxed an additional hour then concentrated to afford a crude mixture, which was purified over silicagel to afford 6 as a 70:30 E:Z mixture of isomers.

6: IR (neat): 3051, 2928, 1565, $1174 \mathrm{~cm}^{-1} ;{ }^{1} \mathrm{H} \mathrm{NMR}\left(\mathrm{CDCl}_{3}, 400 \mathrm{MHz}\right): \delta 2.03$ (quint., $J=6.6 \mathrm{~Hz}$, $2 \mathrm{H}, \mathrm{CH}_{2} \mathrm{CH}_{2} \mathrm{~S}, \mathrm{Z}$ ), 2.10 (quint., $J=6.6 \mathrm{~Hz}, 2 \mathrm{H}, \mathrm{CH}_{2} \mathrm{CH}_{2} \mathrm{~S}, E$ ), 2.87 (tdd, $J=6.6,3.0,1.5 \mathrm{~Hz}, 2 \mathrm{H}$, $\left.=\mathrm{CCH}_{2}, Z\right), 2.98\left(\mathrm{tdd}, J=6.6,3.0,1.8 \mathrm{~Hz}, 2 \mathrm{H},=\mathrm{CCH}_{2}, E\right), 3.05-3.11\left(\mathrm{~m}, 2 \mathrm{H}, \mathrm{SCH}_{2}, E+Z\right), 6.00(\mathrm{bd}$, $J=21.0 \mathrm{~Hz}, 1 \mathrm{H},=\mathrm{CH}, Z), 6.06(\mathrm{dt}, J=21.0 \mathrm{~Hz}, 1.8 \mathrm{~Hz}, 1 \mathrm{H},=\mathrm{CH}, E), 7.41-7.53(\mathrm{~m}, 6 \mathrm{H}$, arom., $E+Z), 7.69-7.80(\mathrm{~m}, 4 \mathrm{H}$, arom., $E+Z) ;{ }^{13} \mathrm{C} \mathrm{NMR}\left(\mathrm{CDCl}_{3}, 100 \mathrm{MHz}\right): \delta 28.4\left(\mathrm{CH}_{2} \mathrm{CH}_{2} \mathrm{~S}, Z\right), 30.7$ $\left(C_{2} \mathrm{CH}_{2} \mathrm{~S}, E\right), 34.3\left(\mathrm{SCH}_{2}, E\right), 35.9\left(\mathrm{SCH}_{2}, Z\right), 36.1\left(\mathrm{~d}, J=19.7 \mathrm{~Hz},=\mathrm{CCH}_{2}, E\right), 42.0(\mathrm{~d}$, $\left.J=14.6 \mathrm{~Hz},=\mathrm{CCH}_{2}, Z\right), 104.4(\mathrm{~d}, J=109.7 \mathrm{~Hz},=\mathrm{CHP}, Z), 105.3(\mathrm{~d}, J=108.0 \mathrm{~Hz},=\mathrm{CHP}, E), 128.4$ (d, $J=10.0 \mathrm{~Hz}, \mathrm{CH}$ arom., Z), 128.5 (d, $J=11.1 \mathrm{~Hz}, \mathrm{CH}$ arom., $E$ ), 130.9 (d, $J=9.4 \mathrm{~Hz}, \mathrm{CH}$ arom., $E$ ), 131.1 (d, $J=10.3 \mathrm{~Hz}, \mathrm{CH}$ arom., Z), 131.5 (bs, CH arom., $E+Z$ ), 133.9 (d, $J=104.5 \mathrm{~Hz},=\mathrm{CP}, Z$ ), $134.7(\mathrm{~d}, J=105.4 \mathrm{~Hz},=\mathrm{CP}, E), 168.8(=\mathrm{CS}, Z), 170.4(\mathrm{~d}, J=5.1 \mathrm{~Hz},=\mathrm{CS}, E) ;{ }^{31} \mathrm{P} \mathrm{NMR}\left(\mathrm{CDCl}_{3}\right.$, $162 \mathrm{MHz}$ ): $\delta 22.2(E), 23.5(Z)$; elemental analysis (\%) for $\mathrm{C}_{17} \mathrm{H}_{17} \mathrm{OPS}$ (300.36): calcd. C 67.98, H 5.70; found C 67.94, H 5.71.

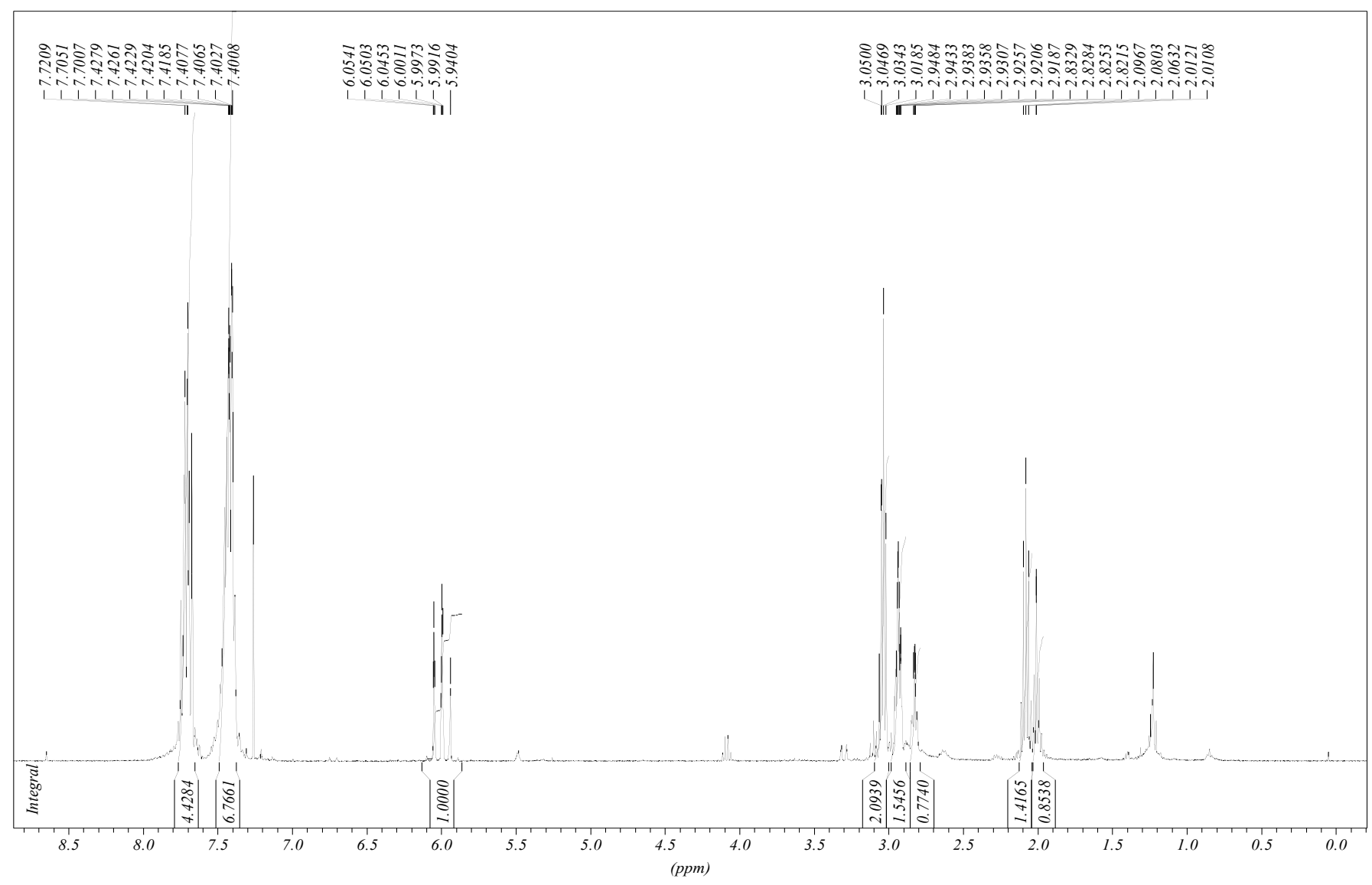




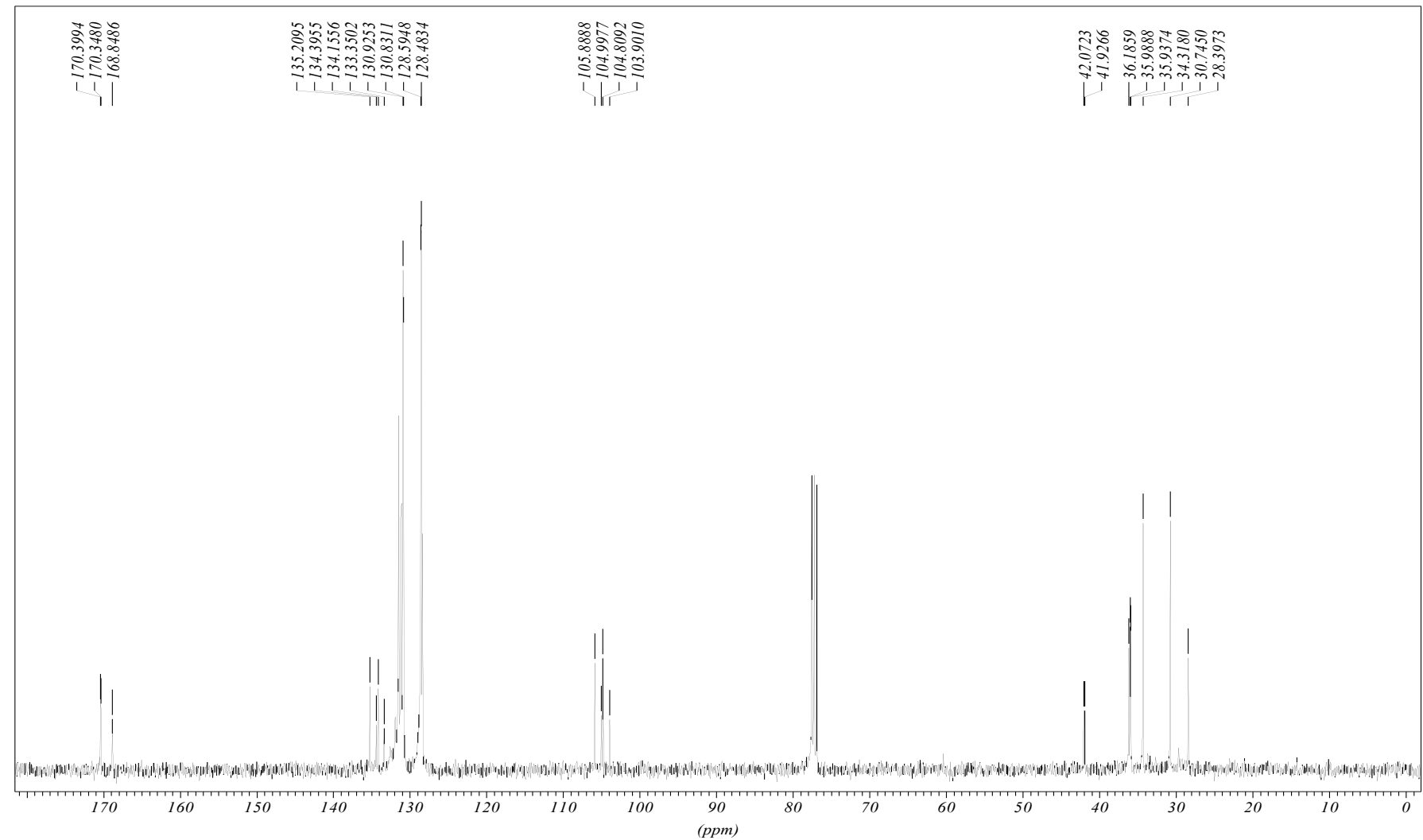




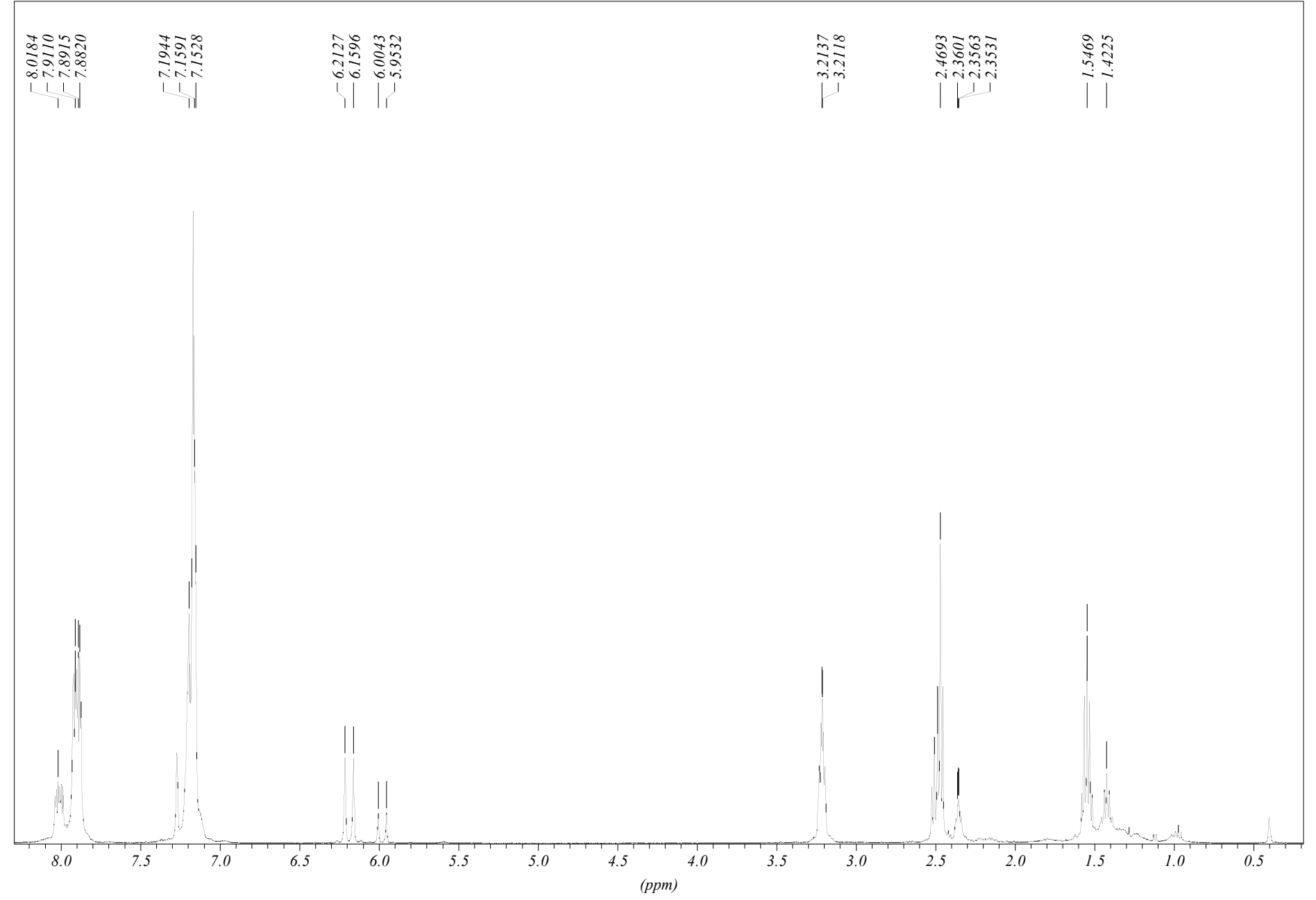

${ }^{1} \mathrm{H}-\mathrm{NMR}$ in $\mathrm{C}_{6} \mathbf{D}_{6}$ 
nOeDIFF: irradiation of $=\mathrm{CCH}_{2}$ in the minor compound (in $\mathrm{C}_{6} \mathrm{D}_{6}$ )

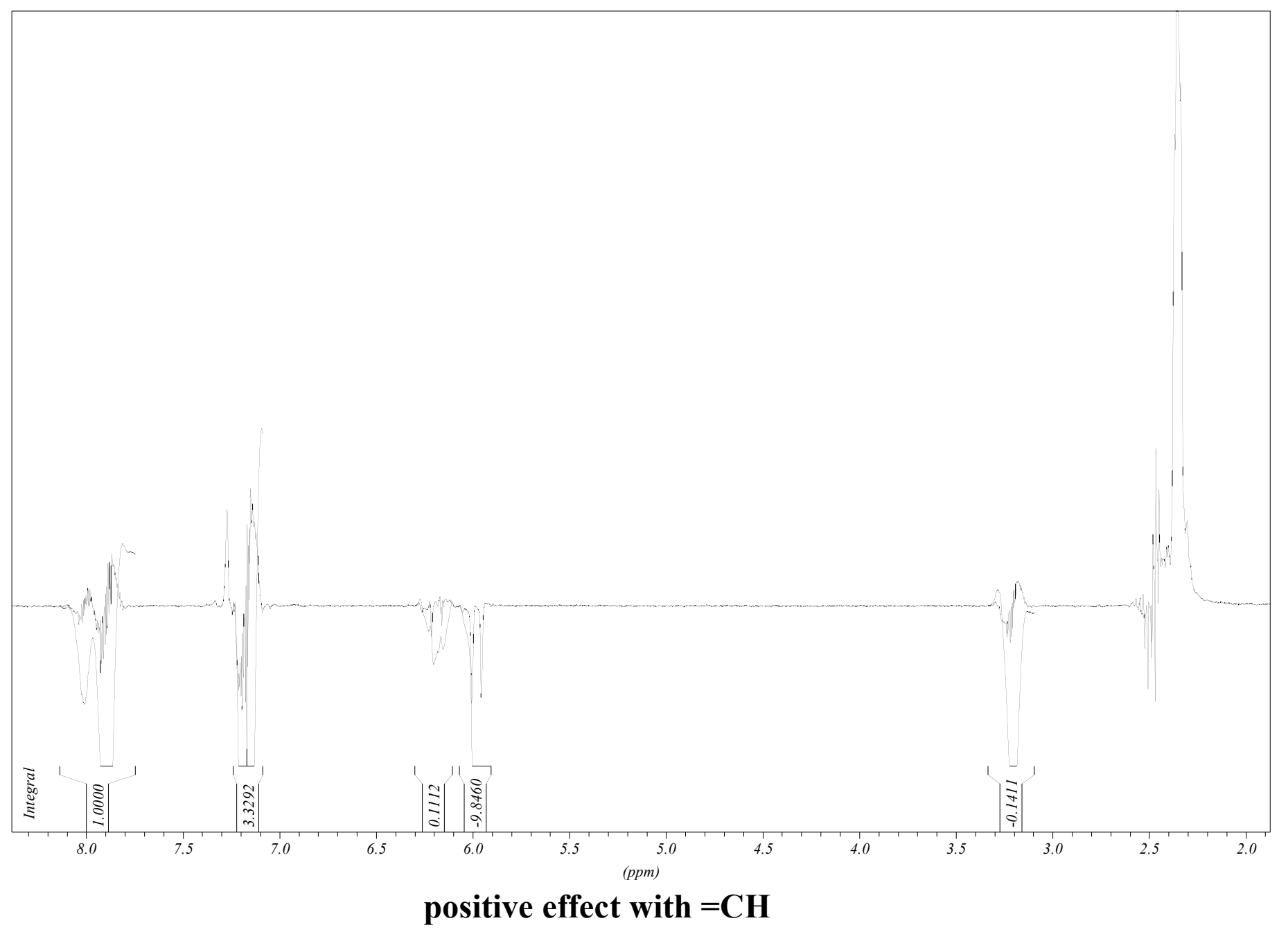


nOeDIFF: irradiation of $=\mathrm{CCH}_{2}$ in the major compound (in $\mathrm{C}_{6} \mathrm{D}_{6}$ )

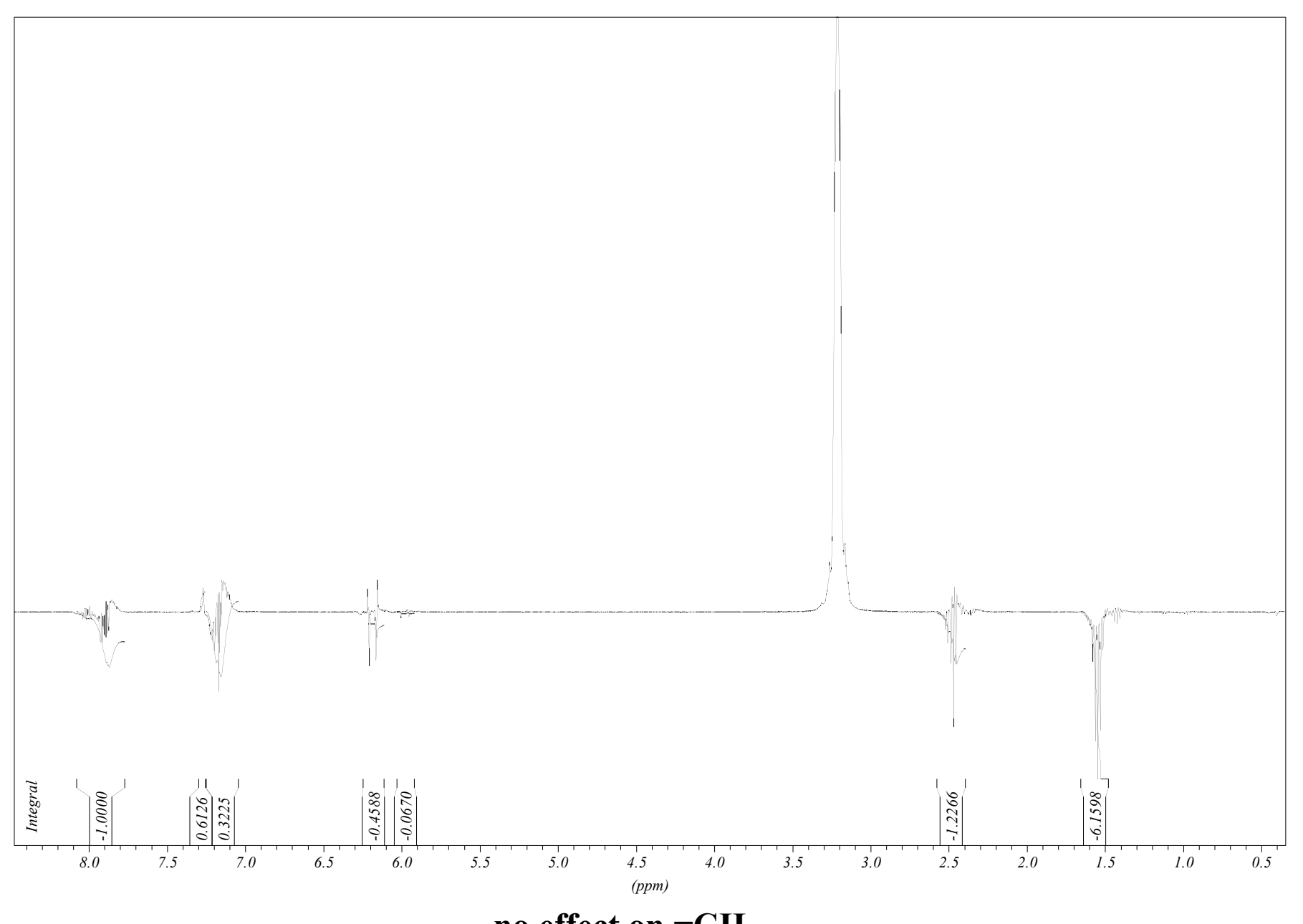

no effect on $=\mathbf{C H}$ 